\title{
The Effects of Acute Neonatal Pain on Expression of Corticotropin-Releasing Hormone and Juvenile Anxiety in a Rodent Model
}

\author{
(D) Jared T. Zuke, ${ }^{1,3}$ Makaela Rice, ${ }^{1}$ Jacob Rudlong, ${ }^{1}$ Taylor Paquin, ${ }^{1}$ Erica Russo, ${ }^{2}$ and ${ }^{\circledR}$ Michael A. \\ Burman $^{1,3}$
}

https://doi.org/10.1523/ENEURO.0162-19.2019

${ }^{1}$ Department of Psychology, University of New England, ${ }^{2}$ Department of Biology, University of New England, and

${ }^{3}$ Center for Excellence in the Neurosciences, University of New England, 11 Hills Beach Rd. Biddeford, ME 04005

\begin{abstract}
Premature infants in the neonatal intensive care unit (NICU) may be subjected to numerous painful procedures without analgesics. One necessary, though acutely painful, procedure is the use of heel lances to monitor blood composition. The current study examined the acute effects of neonatal pain on maternal behavior as well as amygdalar and hypothalamic activation, and the long-term effects of neonatal pain on later-life anxiety-like behavior, using a rodent model. Neonatal manipulations consisted of either painful needle pricks or non-painful tactile stimulation in subjects' left plantar paw surface which occurred four times daily during the first week of life [postnatal day (PND)1-PND7]. Additionally, maternal behaviors in manipulated litters were compared against undisturbed litters via scoring of videotaped interactions to examine the long-term effects of pain on dam-pup interactions. Select subjects underwent neonatal brain collection (PND6) and fluorescent in situ hybridization (FISH) for corticotropin-releasing hormone (CRH) and the immediate early gene c-fos. Other subjects were raised to juvenile age (PND24 and PND25) and underwent innate anxiety testing utilizing an elevated plus maze (EPM) protocol. FISH indicated that neonatal pain influenced amygdalar $\mathrm{CRH}$ and c-fos expression, predominately in males. No significant increase in c-fos or $\mathrm{CRH}$ expression was observed in the hypothalamus. Additionally, neonatal pain altered anxiety behaviors independent of sex, with neonatal pain subjects showing the highest frequency of exploratory behavior. Neonatal manipulations did not alter maternal behaviors. Overall, neonatal pain drives $\mathrm{CRH}$ expression and produces behavioral changes in anxiety that persist until the juvenile stage.
\end{abstract}

Key words: amygdala; anxiety; CRF; neonatal; pain

\section{Significance Statement}

This report expands on current rodent model research performed to assess the long-term effects of highly used neonatal intensive care unit (NICU) procedures. The NICU plays an integral role in pediatric medicine by significantly reducing infant mortality and providing necessary procedures to preterm or unwell newborns. However, procedures in the NICU are often stressful and painful. A common procedure performed in the NICU is heel lances to monitor blood composition. This, along with numerous other painful procedures, are often performed on NICU infants without the benefit of analgesics. Our study identifies key neurologic indicators which are altered in response to neonatal pain. Additionally, we explore the later anxiety of subjects exposed to neonatal pain. 


\section{Introduction}

Premature infants in the neonatal intensive care unit (NICU) may be subjected to numerous painful procedures without analgesics (Anand et al., 1999; Bhutta et al., 2002; Simons et al., 2003; Grunau et al., 2006; Anand, 2007; Carbajal et al., 2008). Exposure to these procedures is correlated with an increased risk of anxiety, mood disorders, and pain sensitivity, later in life (Grunau et al., 2006; Hermann et al., 2006). However, because infants who are in the NICU for longer are likely more ill, it is often unclear whether later behavioral changes are the result of NICU trauma or their original ailment. Rodent models can be used to distinguish between these possibilities via the use of healthy neonates that are subject to manipulations which mimic NICU treatment (Fitzgerald, 1987; Anand et al., 1999; Bhutta et al., 2002; Grunau et al., 2006; Davis et al., 2018).

Neonatal pain has long-term effects on rodents. Rodents have the capacity to perceive noxious stimuli at birth (Fitzgerald, 1987). There is a body of behavioral data which indicates that neonatal rodents exposed to repeated tactile pain exhibit reduced pain thresholds (Anand et al., 1999; Davis et al., 2018; de Carvalho et al., 2019), elevated anxiety in exploratory and social tasks (Anand et al., 1999; Anand and Scalzo, 2000; Schellinck et al., 2003), and a higher preference for alcohol as adults (Anand et al., 1999), although others find no effect on anxiety behavior (Ranger et al., 2018) or a reduction of conditioned freezing (Davis et al., 2018). Importantly, differences in outcome may be due, in part, to the nature of the pain, as studies using neonatal inflammatory pain have found reduced pain sensitivity and lower anxiety in adulthood (Bhutta and Anand, 2001; Anseloni et al., 2005), although other methodological differences likely play a role. Regardless, the neurobiological mechanisms underlying these effects remain largely unknown.

One structure likely to be affected by neonatal pain is the amygdala, which appears to be involved in many forms of negative emotionality, including innate anxiety and pain (Davis, 1992; Janak and Tye, 2015). For exam-

Received May 1, 2019; accepted September 27, 2019; First published October 10, 2019.

The authors declare no competing financial interests.

Author contributions: J.T.Z., M.R., and M.A.B. designed research; J.T.Z., M.R., J.R., and T.P. performed research; J.T.Z., M.R., J.R., T.P., E.R., and M.A.B. analyzed data; J.T.Z., M.R., and M.A.B. wrote the paper.

This work was supported was by the National Institute of General Medical Sciences (NIGMS) Grant P20GM103643, the National Institute of Child Health and Human Development/NIGMS Grant 1R15HD091841 (to M.A.B.), the University of New England (UNE) Summer Undergraduate Research Experience (SURE) Program, and the UNE Peter Morgane Student Research Fellowship.

Acknowledgements: We thank Brandon Buck and Divya Padmanabhan for their contribution to data collection; Seth Davis, PhD, and other members of the Burman Collaborative for procedural and technical assistance; and the UNE COBRE Histology and Imaging Core for their technical expertise.

Correspondence should be addressed to Michael A. Burman at mburman@une.edu.

https://doi.org/10.1523/ENEURO.0162-19.2019

Copyright @ 2019 Zuke et al.

This is an open-access article distributed under the terms of the Creative Commons Attribution 4.0 International license, which permits unrestricted use, distribution and reproduction in any medium provided that the original work is properly attributed. ple, activation of the amygdala, as measured by c-fos expression (Kovács, 2008), is observed after exposure to the elevated plus maze (EPM; Silveira et al., 1993). Physical pain produces a similar activation (Merali et al., 2004). Moreover, the amygdala receives input regarding aversive stimuli and projects to regions controlling descending pain inhibition pathways, anxious and defensive behaviors, and autonomic activation. The current studies examined the effects of neonatal pain on amygdalar c-fos expression and EPM behavior.

There is also evidence that corticotropin-releasing hormone $(\mathrm{CRH})$ may be involved in the long-term consequences of neonatal pain. $\mathrm{CRH}$ is produced in several densely populated regions of the CNS, including the central nucleus of the amygdala $(\mathrm{CeA})$, paraventricular nucleus of the hypothalamus (PVN), and bed nucleus of the stria terminalis (BNST; Holsboer, 1999; Plotsky et al., 2005). Amygdalar $\mathrm{CRH}$ has increasingly been implicated in many forms of stress (Cratty et al., 1995; Korte and De Boer, 2003). Additionally, other studies have indicated that chronic pain (Ulrich-Lai et al., 2006) and neonatal stress (Brunson et al., 2001) elevate amygdalar, but not hypothalamic, $\mathrm{CRH}$ expression. Thus, the current experiments examine amygdalar $\mathrm{CRH}$ expression following neonatal pain.

The purpose of this study was to further our understanding of neuronal mechanisms by which neonatal pain affects later emotional responding. Additionally, our study assessed whether there were lasting effects of neonatal pain and handling on maternal behaviors. We hypothesized that our neonatal pain model would cause an increased expression of $\mathrm{CRH}$ mRNA in the amygdala of pups independent of maternal rearing behaviors. Furthermore, we hypothesized that downstream effects of neonatal pain would alter juvenile innate anxiety responses. To accomplish this, the current studies used c-fos and $\mathrm{CRH}$ fluorescent in situ hybridization (FISH) as a measure of nociceptive neuronal activation and $\mathrm{CRH}$ expression and the EPM to measure juvenile innate anxiety.

\section{Materials and Methods}

\section{Subjects}

Most of the subjects were bred in house from Charles River Sprague Dawley stock. These subjects were used for maternal behavior recordings, neonatal FISH, and anxiety assays. A subset of subjects was ordered as timedpregnant dams (Charles River; used for anxiety assays) before the development of our breeding colony. Timedpregnant females arrived on gestational day (GD)12, were singly housed, and gave birth in house on either GD21 or GD22. Our in-house breeding procedure was similar to other published works (Davis et al., 2018; Ogilvie and Rivier, 1997) and also all gave birth, singly housed on either GD21 or GD22. GD22 was considered the day of birth and called postnatal day (PND)0. Statistical analysis found no significant differences between timed-pregnant and in-house bred subjects and they were therefore combined for all further analyses.

Starting at birth, all rats were housed in $43 \times 44 \times 20$ cm closed-environment cages (Innovive). Rats were main- 


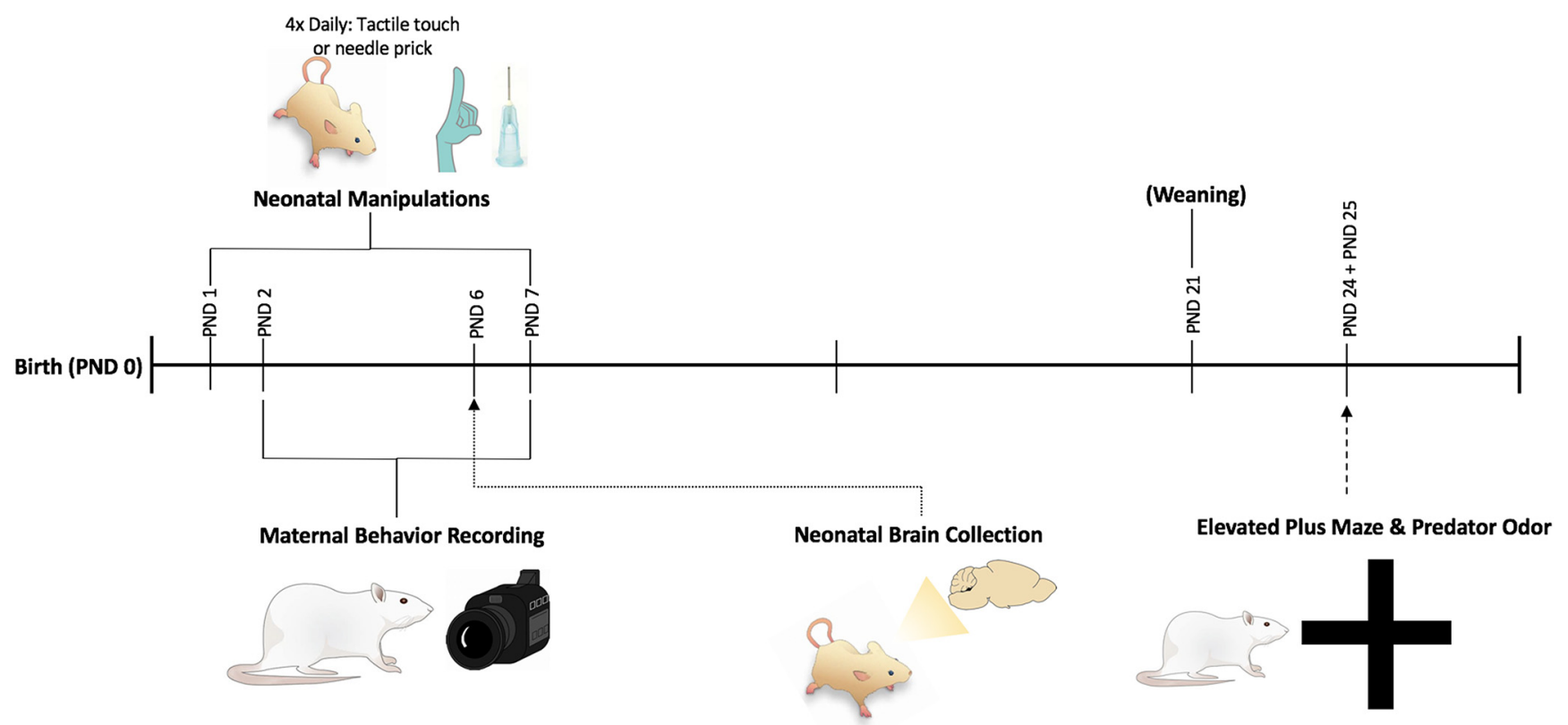

Figure 1. Timeline representation of animal experimentation process from birth to elevated plus maze behavioral testing. Neonatal manipulations were repeated four times daily, $2 \mathrm{~h}$ apart. Manipulations entailed either a tactile touch or needle prick of the left hindpaw PND1-PND7. Maternal behavior recordings occurred before the start of manipulations PND2-PND7. A subset of subjects from litters were euthanized and underwent brain collection on PND6 for FISH. Weaning of litters into same sex groupings occurred on PND21. Elevated plus maze and predator odor exposure occurred on PND24 and PND25 in a counterbalanced order by day.

tained on 12/12 h light/dark cycle with lights on at 7 A.M. and were provided food and water ad libitum.

On PND1, litters were randomly assigned to either neonatal manipulation or an undisturbed control condition, with every litter cohort containing at least one litter of each assignment. Litters were culled to 10 rats (five males and five females when possible). Litters were weaned on PND21 and housed with same-sex littermates containing approximately five rats per cage. On completion of anxiety assays, rats were euthanized by $\mathrm{CO}_{2}$ asphyxiation. All rats used for FISH were euthanized immediately before neurologic tissue collection via SomnaSol pentobarbital (Henry Schein Animal Health) overdose and cardiac perfusion. All animal euthanasia was consistent with American veterinary association procedures.

A total of 16 litters were recorded for maternal behavior analysis (8 neonatal manipulation and 8 undisturbed). For RNAscope FISH experimentation, 35 rats from seven neonatally-manipulated litters and six undisturbed litters were used. Lastly, 107 rats from 13 neonatally-manipulated litters and 11 undisturbed litters were used in our anxiety assays. In all cases, no more than one same-sex littermate was assigned to any experimental condition. All animal procedures were performed in accordance with University of New England Institutional Animal Care and Use Committee and in compliance with $\mathrm{NIH}$ animal care and use guidelines.

\section{Neonatal manipulations}

Similar to manipulation procedures performed by (Davis et al., 2018; Anand et al., 1999), pups in litters designated for neonatal manipulation were marked and randomly assigned to receive either a needle prick from a 23-gauge needle tip in their left hindpaw (pain group), or a noninvasive tactile touch on the left-hind paw by the experimenter (handled group). All neonatal manipulation litters had both groups equally represented. Manipulations occurred four times per day, $2 \mathrm{~h}$ apart from PND1 to PND7, the exception being subjects used for FISH, which were euthanized for neurologic tissue collection on PND6, 15 min after the first manipulation period on that day see Figure 1 for experimental timeline.

\section{Maternal behavior}

Litters randomly designated for maternal behavior observation were recorded using two GoPro HD Hero Session cameras for $1 \mathrm{~h}$ before the first manipulation on PND2-PND7, to examine the long-term effects of neonatal pain on dam-pup interactions in the absence of the acute effects of handling. Home cages were removed from the rack and placed on a cart with one camera mounted beside the cage and another mounted above (two camera angles per litter). Recordings for both neonatal manipulation and undisturbed litters were done simultaneously on each day of recording. Video recordings were later observed and scored by trained experimenters using Behavioral Observation Research Interactive Software (BORIS; Friard and Gamba, 2016). Scored behaviors were similar to other rodent maternal behavior studies (Caldji et al., 1998; Walker et al., 2003; LaPrairie and Murphy, 2007). These behaviors were pup licking/grooming, nursing (both passive and arched-back nursing), nest building (including burrowing), maternal self-grooming, and maternal contact (any dam to pup physical contact including licking/grooming and/or nursing). Our behavior scoring methods were adapted from Rincel et al. (2016), 


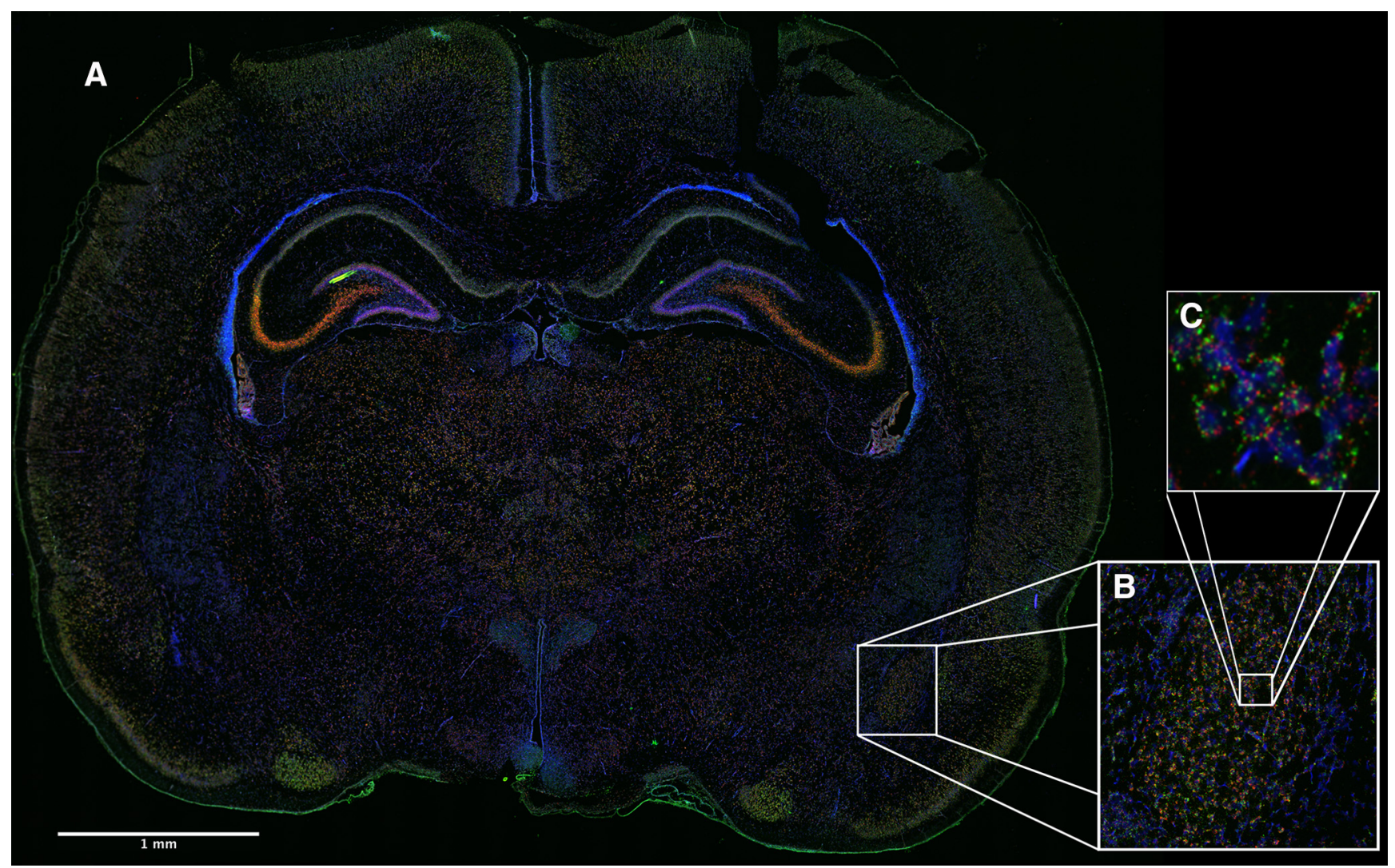

Figure 2. Optimized positive control section of RNAscope FISH in a PND6 rat brain. Green (channel 1): staining Polr2a, red (channel 2): staining PPIB, blue (DAPI). (A) complete coronal section, $(\boldsymbol{B})$ zoomed in section of the amygdala, $(\boldsymbol{C})$ zoomed in region of the amygdala to demonstrate punctate staining pattern.

with behaviors scored for $10 \mathrm{~s}$ at the start of every 2-min interval of recorded observation, thus totaling 30 observation periods for every $1-\mathrm{h}$ video. Lastly, $\sim 10 \%$ of videos were scored by two experimenters with resulting interrater reliability correlations of $0.803^{a}$ for pup licking/grooming, $0.921^{\mathrm{b}}$ for nursing, $0.941^{\mathrm{c}}$ nest building, $0.969^{\mathrm{d}}$ maternal self-grooming, and $0.936^{\mathrm{e}}$ maternal contact.

\section{Fluorescence in situ hybridization}

FISH was conducted using a commercially available system [RNAscope; Advanced Cell Diagnostics (ACD)]. This technique assesses mRNA expression in a quantifiable manner. First, neonatal brain tissue was collected following intra-cardiac perfusion and post-fixed in Bouin's fixative solution (RICCA Chem.) for $2 \mathrm{~h}$ before cryoprotection and refrigeration in a $30 \%$ sucrose solution. Subsequently, brains were embedded in Tissue-Tek O.C.T compound (Sakura Finetek) and flash frozen using liquid nitrogen. Brains were cryosectioned into $15-\mu \mathrm{m}$ sections and mounted onto Superfrost plus microscope slides (VWR). Tissue sections were stored for approximately one month at $-80^{\circ} \mathrm{C}$ before undergoing the FISH procedure.

The FISH used probes targeting $\mathrm{CRH}$ (product number: 318931) and c-fos (product number: 403591-C2). The protocol was developed using RNAscope Multiplex Fluorescent Reagent Kit v2 user manual (document number: 323100-USM), manufacturer technical note regarding tissue detachment, and manufacturer modifications for fixed frozen tissue (ACD). In the final steps of the protocol, DAPI was applied to brain sections as a counterstain for amygdala and hypothalamic regional identification.

RNAscope assays were conducted in batches consisting of tissue collected on the same date from the same litters to limit variance in animal life experience, tissue treatment, and target degradation variability. Tissue from all three conditions (pain, handled, and undisturbed) and both sexes were included in each batch. Each batch also contained additional tissue processed with either a positive control probe [product number: 320891; containing Polr2a (channel 1), PPIB (channel 2)] or negative control probe [product number: 320871; containing DapB gene accession EF191515 from the SMY strain of Bacillus subtilis (channels 1 and 2)]. Per manufacturer instructions (RNAscope; ACD), these controls were to ensure consistency and procedural accuracy between subsequent FISH batches. See (Fig. 2) for optimized positive control image example. All negative probe images demonstrated no specific staining.

Fluorescence multiplex imaging was done between $2 \mathrm{~d}$ and two weeks after FISH. All images for quantification were taken under the same magnification $(20 \times)$ and exposure settings (1/25 s) for quantified channels $(\mathrm{CRH}$ and c-fos) on a Keyence BZ-X710 microscope. The DAPI channel exposures varied somewhat between sections due to the inherent variability in DAPI application. Sections were overlaid and stitched for analysis using image 


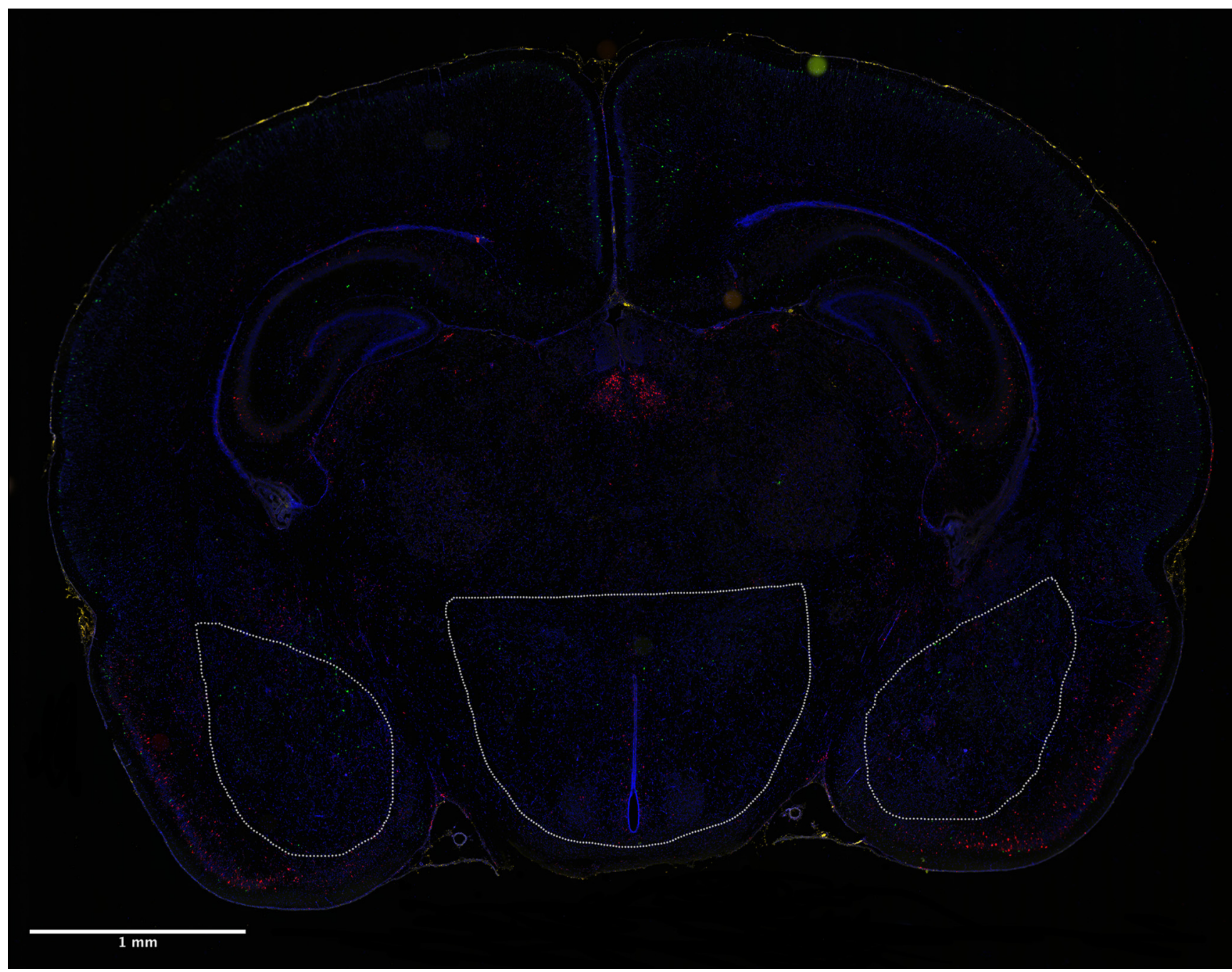

Figure 3. Representative coronal brain section depicting analyzed regions of interest (amygdalae and hypothalamus). Subject depicted is a male from the pain condition. CRH (green), c-fos (red), DAPI (blue).

merge software provided with Keyence BZ-X710 microscope (Keyence). See Figure 3 for a sample picture with identified regions of interest.

Images were analyzed using the FIJl package of NIH's open source image analysis software ImageJ (Schindelin et al., 2012; Schneider et al., 2012). Overlaid images were first subject to color separation and the DAPI channel was used to identify amygdala and hypothalamic regions of interest. $\mathrm{CRH}$ and $\mathrm{c}$-fos expression were measured in our regions of interest for percentage area expressing the signal (percentage area), expression intensity (measured by Otsu threshold grayscale values), and instances of expression colocalization.

Values from the right and left regions of interest in individual brain sections were combined to generate a value of the average percentage area expressing the signal (percentage area), average expression intensity (intensity), and summation of colocalization occurrences (colocalization) for each subject. Further, a "baseline value" was calculated by taking the average of percentage area for undisturbed animals of each sex. This baseline value was then used to calculate a percentage difference score from undisturbed in both sexes via the equation: (area expressing target probe in the subject baseline value)/baseline value $\times 100$. The resulting values were referred to as percentage difference in area. This was done for both $\mathrm{CRH}$ and $\mathrm{c}$-fos expression in both the amygdala and hypothalamus.

\section{Anxiety assays}

After weaning (on PND24 and PND25), subjects were removed from their home cage, weighed, and placed in individual transport cages. Subjects were transported to the testing room and underwent a 10-min habituation period before behavioral testing to assess innate anxiety levels using an EPM and predator odor exposure in a counterbalanced by day manner.

\section{Elevated Plus Maze}

Our EPM apparatus comprised two open arms $(51 \times 10$ $\mathrm{cm})$ and two closed arms $(51 \times 10 \mathrm{~cm})$ connected via a center platform $(10 \times 10 \mathrm{~cm})$. The apparatus was $49.5 \mathrm{~cm}$ above floor level, and constructed from wood. The only lighting in the room was a dimly lit, incandescent floor 
standing lamp. Before each subject was tested, the EPM was cleaned with $70 \% \mathrm{EtOH}$. Subjects were placed in the center of the apparatus facing an open arm and were allowed to explore freely for $5 \mathrm{~min}$. The experimenter was outside of the testing room for the duration of the test. Activity was recorded and tracked using EthoVision XT (Noldus) software. Four measures were assessed: openarm entries, open-arm time, total distance traveled, and total arm entries.

\section{Predator odor}

Filter paper containing either two drops of $1 \%$ peppermint oil or $\sim 0.1-\mathrm{ml}$ red fox urine (trap shack company; via Amazon.com) was placed inside an open Petri dish, and subsequently placed on opposite sides of a vent toped $(44 \times 23.5 \mathrm{~cm})$ shoebox cage. The shoebox cage was placed inside a fume hood with the fan and lights on during the duration of the test. Rats were placed in the center of the cage facing away from the experimenter, and a real-time log of the subject's activity was recorded for $10 \mathrm{~min}$. The cage was cleaned with sparkleen 1 (Fisher Scientific) between each test. Fox urine was naturally derived and stored at room temperature in an air tight container (in lab) when not in use.

Despite pilot data to the contrary, all rats demonstrated a common strong side preference (likely due to some unknown characteristic of the fume hood) rather than a specific reaction to the odors. Therefore, the predator odor experimental data were not analyzed further, although the EPM data from these rats was retained.

\section{Statistical analysis}

All data (maternal behavior, FISH, and anxiety assays) were analyzed with IBM SPSS version 22 mixed model ANOVAs and Tukey's post hoc analysis were performed where applicable (See Table 1). Maternal behavior statistical analysis consisted of 2 (litter type: neonatal manipulation, undisturbed) $\times 6$ (PND2-PND6) repeated measures MANOVAs with testing day serving as the repeated variable.

RNAscope FISH statistical analysis consisted of first a 2 (amygdala side: right, left) $\times 2$ (sex: male, female) $\times 3$ (neonatal condition: undisturbed, handled, and pain) ANOVA to assess for staining lateralization in the $\mathrm{CRH}$ channel. When no staining lateralization was found, 2 (sex: male, female) $\times 3$ (neonatal condition: undisturbed, handled, and pain) ANOVAs were conducted for each measure regarding $\mathrm{CRH}$ and $\mathrm{c}$-fos (percentage difference in area, sum of colocalization, and Otsu threshold grayscale value). As sex differences were common, follow-up one-way ANOVAs and Tukey's HSD post hoc tests were used. Guidance from ACD suggests that number of positive straining events (operationalized here as area), rather than brightness, is the best method to assess differences in RNAscope expression.

Statistical analysis for anxiety assays consisted of a 2 (testing order: predator exposure on PND24, PND25) $\times 2$ (sex: male, female) $\times 2$ (breeding condition) $\times 3$ (neonatal condition: undisturbed, handled, pain) ANOVA comparing average time in open arms, number of open arm entries, number of total arm entries, and total distance traveled across each group. Follow-up ANOVAs and Tukey's post hoc tests were used as appropriate.

\section{Results}

\section{Experiment 1: maternal behavior}

Dams of litters that experienced neonatal manipulations and dams of undisturbed litters did not differ in their behaviors toward pups as shown by 2 (litter type: neonatal manipulation, undisturbed) $\times 6$ (day: PND2-PND7) repeated measures MANOVAs with testing day serving as the repeated variable. Condition alone had no effect on any maternal behavior measure including the following: pup licking/grooming $F_{(1,14)}=0.170, p=0.686$, partial $\eta^{2}$ $=0.012^{f}$; nursing $F_{(1,14)}=0.343, p=0.568$, partial $\eta^{2}=$ $0.024 \times g$; nest building $F_{(1,14)}=2.14, p=0.166$, partial $\eta^{2}=0.133^{\text {h}}$; maternal self-grooming $F_{(1,14)}=0.604, p=$ 0.450 , partial $\eta^{2}=0.041^{\mathrm{i}}$; and maternal contact $F_{(1,14)}=$ $0.230, p=0.639$, partial $\eta^{2}=0.016^{\mathrm{j}}$.

There was a significant condition $\times$ day interaction for nest building $F_{(5,10)}=3.37, p=0.048$, partial $\eta^{2}=0.628^{k}$ suggesting that time spent nest building differed in the neonatal manipulation litters compared to undisturbed litters (Fig. 4). Subsequent $t$ tests examining the effect of condition on each day found a significant difference on PND2 $p=0.006 ; \mathrm{Cl}=1.821-9.179^{\prime}$ and $a$ trend toward a significant difference on PND7 $p=0.083 ; \mathrm{Cl}=-4.837$ $-0.337^{\mathrm{m}}$; with neonatal manipulation litters having more nest building on both days (Fig. 4). In addition, there was a main effect of day for nursing $F_{(5,10)}=7.06, p=0.005$, partial $\eta^{2}=0.779^{\text {n }}$; nest building $F_{(5,10)}=24.46, p<$ 0.001 , partial $\eta^{2}=0.924^{\circ}$; and maternal interaction $F_{(5,10)}$ $=4.98, p=0.015$, partial $\eta^{2}=0.714^{\mathrm{p}}$. These data suggest that maternal behaviors change over development but are not affected by our acute repeated neonatal manipulations.

\section{Experiment 2: Fluorescence in situ hybridization}

Two subjects (male undisturbed and female handled) were excluded as statistical outliers for having $\mathrm{CRH}$ expression data $>2.5$ SDs from their respective group means in the amygdala. For the hypothalamus experiments, two subjects (male handled and female handled) were excluded for having $\mathrm{CRH}$ expression data $>2.5$ SDs from their respective group means.

There were no observed differences (sex, condition, or interaction) in Otsu threshold mean grayscale values for all measures of $\mathrm{CRH}$ and c-fos in amygdalar and hypothalamic regions (Fig. 5). These finding are consistent with standard RNAscope quantification procedures.

Unexpectedly, sex differences were apparent both in the pattern of results and in most statistical analyses. Therefore, we separated all subsequent analyses for this experiment by sex.

\section{Lateralization of the amygdala}

An initial 2 (amygdala side: right, left) $\times 2$ (sex: male, female) $\times 3$ (neonatal condition: undisturbed, handled, and pain) ANOVA resulted in a significant main effect of hemisphere $F_{(1,27)}=7.66, p=0.010$, partial $\eta^{2}=0.221^{q}$ 
Table 1. Statistical table defining type of test and observed power for each statistical analysis

\begin{tabular}{|c|c|c|c|}
\hline Test & $\begin{array}{l}\text { Data } \\
\text { structure }\end{array}$ & $\begin{array}{l}\text { Type } \\
\text { of test }\end{array}$ & $\begin{array}{c}\text { Power or } \\
\text { confidence interval } \\
\text { (lower/upper) }\end{array}$ \\
\hline a & Presumed normal & Within item interclass correlation; interrater reliability test & Lower: 0.67/upper: 0.88 \\
\hline C & Presumed normal & Within item interclass correlation; interrater reliability test & Lower: 0.90/upper: 0.96 \\
\hline d & Presumed normal & Within item interclass correlation; interrater reliability test & Lower: 0.95/upper: 0.98 \\
\hline e & Presumed normal & Within item interclass correlation; interrater reliability test & Lower: 0.89/upper: 0.96 \\
\hline $\mathrm{h}$ & Presumed normal & $2 \times 6$ repeated measures MANOVA & Power: 0.276 \\
\hline i & Presumed normal & $2 \times 6$ repeated measures MANOVA & Power: 0.112 \\
\hline j & Presumed normal & $2 \times 6$ repeated measures MANOVA & Power: 0.073 \\
\hline k & Presumed normal & $2 \times 6$ repeated measures MANOVA & Power: 0.680 \\
\hline I & Presumed normal & $2 \times 6$ repeated measures MANOVA; $t$ test for condition by day & Lower: 1.82/upper: 9.18 \\
\hline $\mathrm{m}$ & Presumed normal & $2 \times 6$ repeated measures MANOVA; $t$ test for condition by day & Lower: -4.84/upper: 0.34 \\
\hline r & Presumed normal & $2 \times 2 \times 3$ ANOVA & Power: 0.451 \\
\hline s & Presumed normal & $2 \times 2 \times 3$ ANOVA & Power: 0.080 \\
\hline $\mathrm{t}$ & Presumed normal & $2 \times 2 \times 3$ ANOVA & Power: 0.210 \\
\hline $\mathrm{u}$ & Presumed normal & $2 \times 2 \times 3$ ANOVA & Power: 0.353 \\
\hline $\mathrm{v}$ & Presumed normal & $2 \times 2 \times 3$ ANOVA & Power: 0.058 \\
\hline w & Presumed normal & $2 \times 2 \times 3$ ANOVA & Power: 0.088 \\
\hline$x$ & Presumed normal & $2 \times 2 \times 3$ ANOVA & Power: 0.119 \\
\hline y & Presumed normal & ANOVA & Power: 0.704 \\
\hline$z$ & Presumed normal & $2 \times 3$ ANOVA & Power: 0.385 \\
\hline aa & Presumed normal & $2 \times 3$ ANOVA & Power: 0.474 \\
\hline$a b$ & Presumed normal & $2 \times 3$ ANOVA & Power: 0.486 \\
\hline ac & Presumed normal & $2 \times 3$ ANOVA & Power: 0.714 \\
\hline $\mathrm{ad}$ & Presumed normal & $2 \times 3$ ANOVA & Power: 0.455 \\
\hline am & Presumed normal & $2 \times 3$ ANOVA; Tukey's post hoc & Lower: 0.386/upper: 40.01 \\
\hline an & Presumed normal & $2 \times 3$ ANOVA; Tukey's post hoc & Lower: -2.35/upper: 35.51 \\
\hline ao & Presumed normal & $2 \times 3$ ANOVA & Power: 0.423 \\
\hline ap & Presumed normal & $2 \times 3$ ANOVA & Power: 0.390 \\
\hline aq & Presumed normal & $2 \times 3$ ANOVA & Power: 0.071 \\
\hline $\operatorname{ar}$ & Presumed normal & $2 \times 3$ ANOVA & Power: 0.053 \\
\hline as & Presumed normal & $2 \times 3$ ANOVA & Power: 0.110 \\
\hline at & Presumed normal & ANOVA; Tukey's post hoc & Lower: 8.52/upper: 123.84 \\
\hline $\mathrm{au}$ & Presumed normal & ANOVA; Tukey's post hoc & Lower: -10.84/upper: 92.30 \\
\hline av & Presumed normal & ANOVA & Power: 0.139 \\
\hline aw & Presumed normal & ANOVA & Power: 0.348 \\
\hline ax & Presumed normal & ANOVA & Power: 0.157 \\
\hline ay & Presumed normal & ANOVA & Power: 0.999 \\
\hline az & Presumed normal & ANOVA; Tukey's post hoc & Lower: 16.90/upper: 46.76 \\
\hline $\mathrm{ba}$ & Presumed normal & ANOVA; Tukey's post hoc & Lower: 14.64/upper: 48.03 \\
\hline $\mathrm{bb}$ & Presumed normal & ANOVA & Power: 0.106 \\
\hline bc & Presumed normal & ANOVA & Power: 0.133 \\
\hline bd & Presumed normal & ANOVA & Power: 0.094 \\
\hline be & Presumed normal & ANOVA & Power: 0.061 \\
\hline bf & Presumed normal & ANOVA & Power: 0.071 \\
\hline bg & Presumed normal & ANOVA & Power: 0.064 \\
\hline bh & Presumed normal & ANOVA & Power: 0.143 \\
\hline \multicolumn{4}{|c|}{ (Continued) } \\
\hline
\end{tabular}


Table 1. Continued

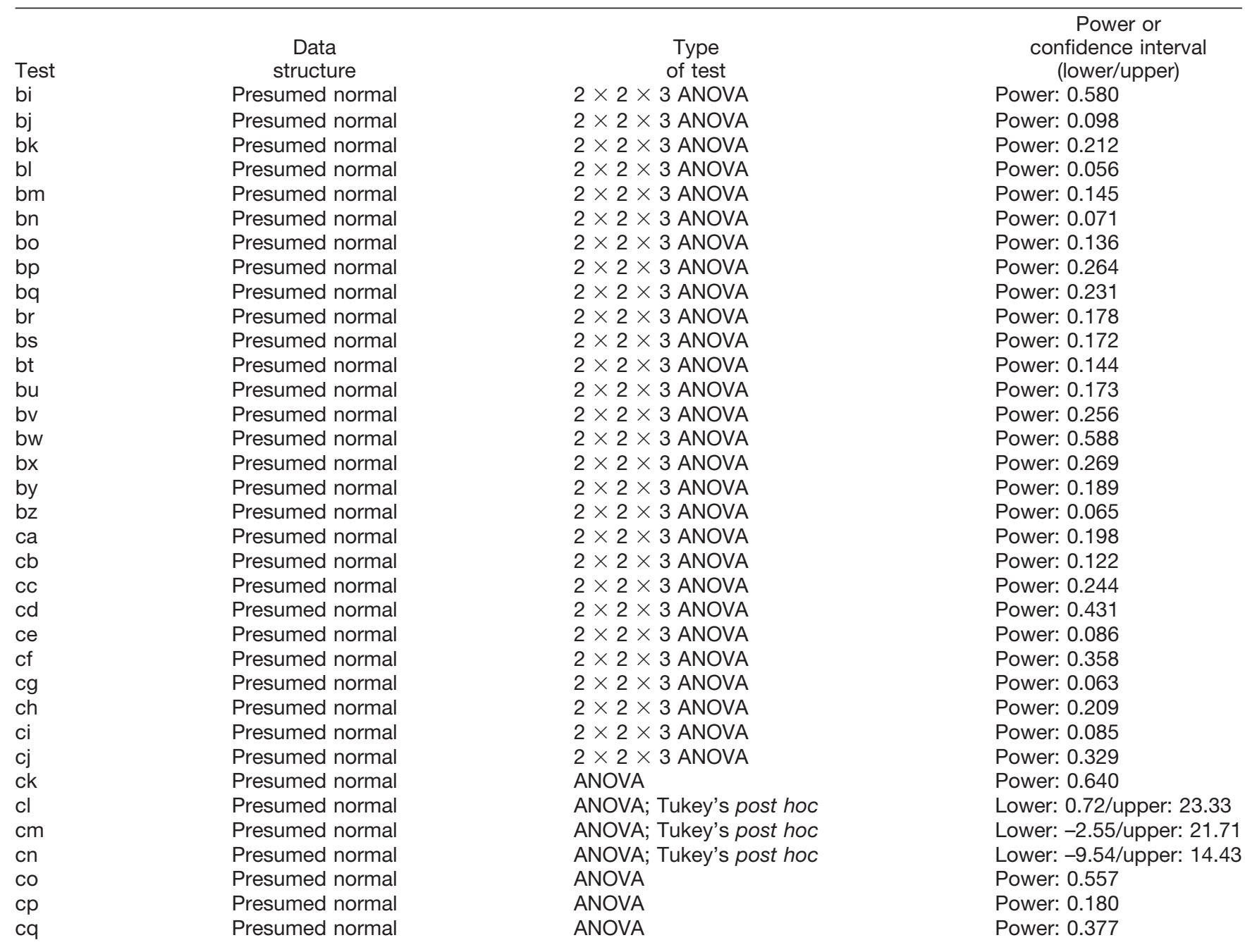

Statistical data pertaining to each test is indicated by superscript letters within text.
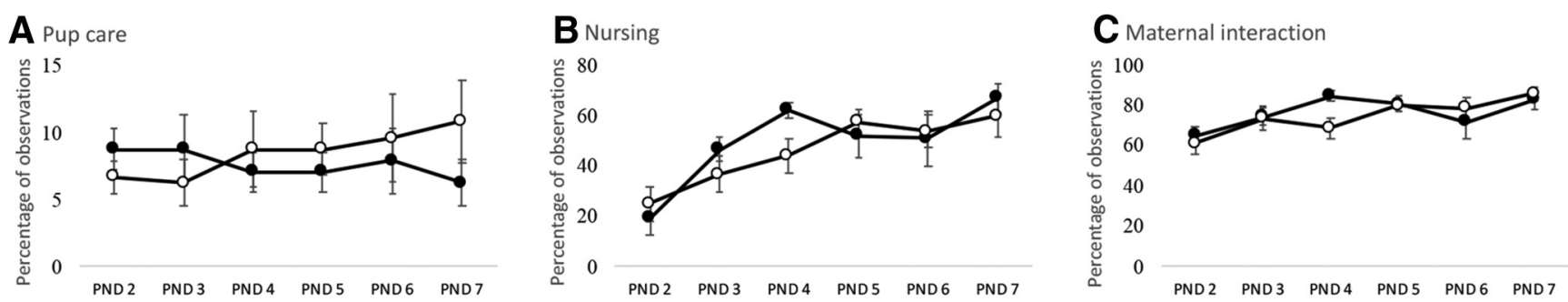

D Nest building

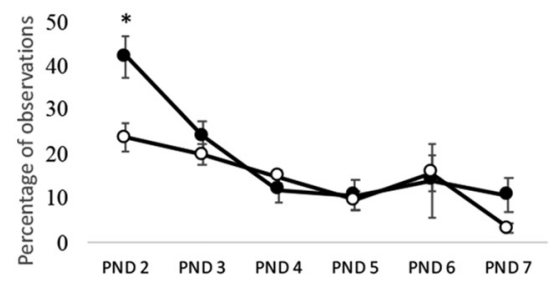

E Self-grooming
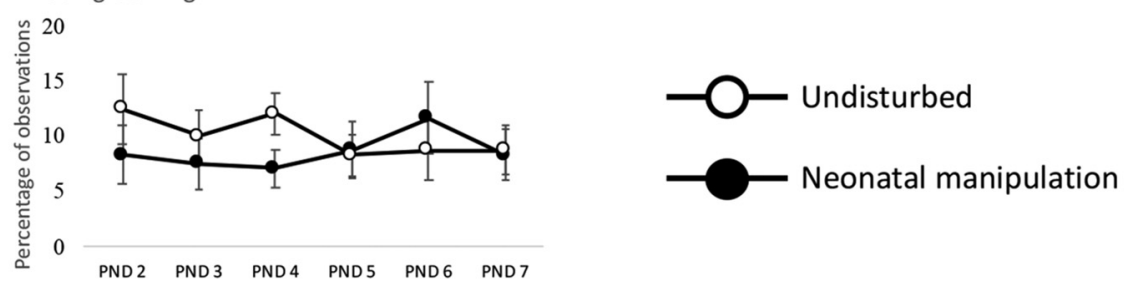

Figure 4. Maternal behaviors recorded from PND2 to PND7 for undisturbed and manipulation litters. Each data point represents the mean of eight dams/litters for percentage of observed maternal behaviors. Recorded behaviors were $(\boldsymbol{A})$ pup care, $(\boldsymbol{B})$ nursing, $(\boldsymbol{C})$ maternal interaction, $(\boldsymbol{D})$ nest building, and $(\boldsymbol{E})$ self-grooming. Error bars represent $\pm \mathrm{SEM}$; $*$ denotes significant $(p<0.05)$ difference between neonatal manipulation and undisturbed litters for that day and behavior. 
A Amygdalar $\mathrm{CRH}$

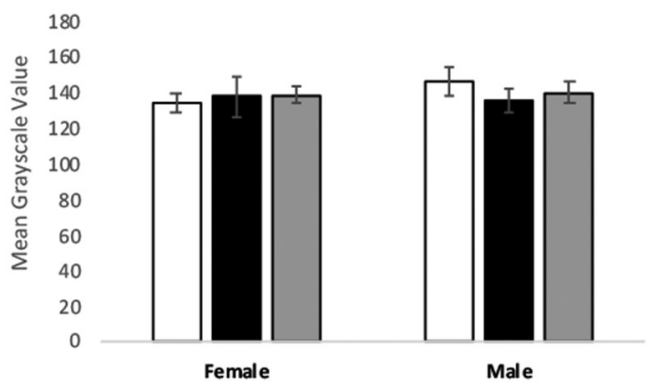

C Hypothalamic $\mathrm{CRH}$

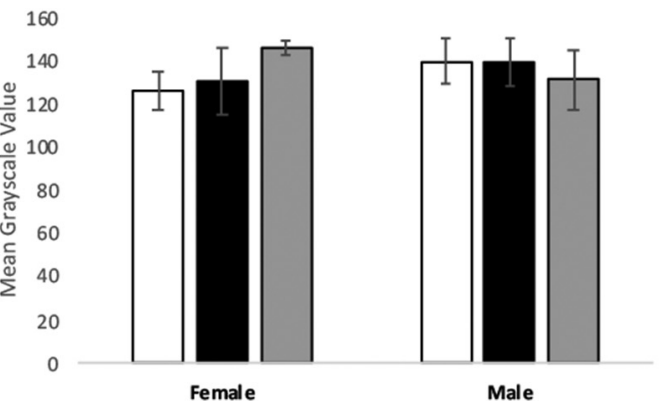

B Amygdalar c-fos

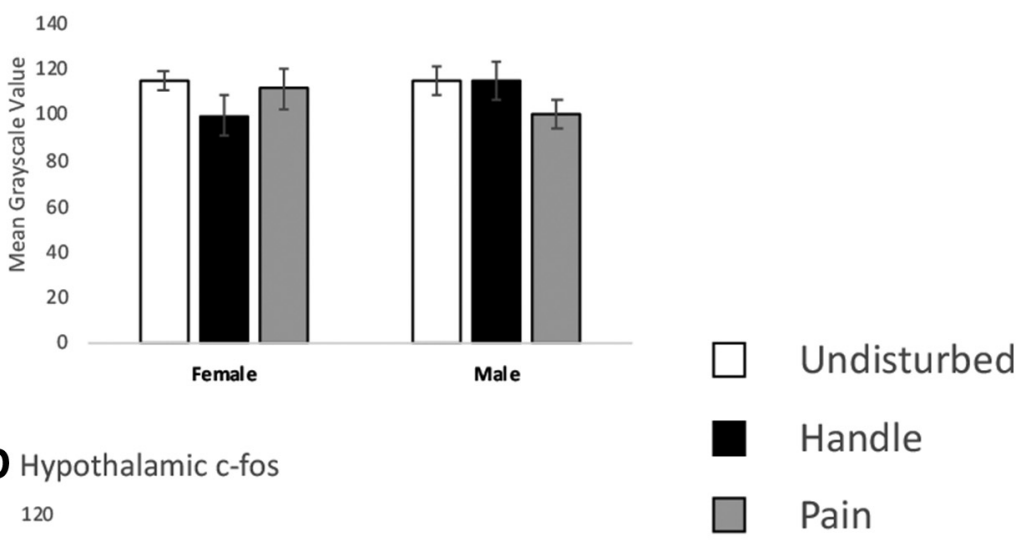

Figure 5. Amygdalar and hypothalamic FISH luminance measures for $\mathrm{CRH}$ and c-fos in PND6 rats. Luminance was measured for all regions via mean grayscale value after Otsu threshold application. All regions and corresponding subjects used in our $\mathrm{CRH}$ and $\mathrm{c}$-fos percentage difference in area measurements were also used in these luminance measurements. Average greyscale values are shown for: $\boldsymbol{A}$ CRH expression in the amygdala, $\boldsymbol{B}$ c-fos expression in the amygdala, $\boldsymbol{C}$ CRH expression in the hypothalalmus, $\boldsymbol{D}$ c-fos expression in the hypothalamus. Data are presented as means with error bars as \pm SEM.

for percentage area expressing $\mathrm{CRH}$. The left amygdala (mean $=0.101$, SEM $=0.006$ ) exhibiting a greater area expressing $\mathrm{CRH}$ compared to right (mean $=0.085$, SEM $=0.005)$. Additionally, we observed a trend toward an interaction between hemisphere $\times \operatorname{sex} F_{(1,27)}=3.63, p=$ 0.068 , partial $\eta^{2}=0.118^{r}$ for percentage area expressing $\mathrm{CRH}$. Male left amygdala (mean $=0.105, \mathrm{SEM}=0.010$ ) showing the highest percentage area expressing $\mathrm{CRH}$ followed by female left amygdala (mean $=0.096$, SEM $=$ 0.009), female right amygdala (mean $=0.091$, SEM $=0.008$ ), and male right amygdala (mean $=0.079$, SEM $=0.007$ ). There were no significant hemisphere $\times$ condition interaction $F_{(2,27)}=0.211, p=0.811$, partial $\eta^{2}$ $=0.015^{\mathrm{s}}$ nor a hemisphere $\times$ sex $\times$ condition interaction $F_{(2,27)}=1.026, p=0.372$, partial $\eta^{2}=0.071^{\mathrm{t}}$ for percentage area expressing $\mathrm{CRH}$.

Additionally, a 2 (amygdala side: right, left) $\times 2$ (sex: male, female) $\times 3$ (neonatal condition: undisturbed, handled, and pain) ANOVA found no main effects or interactions in the lateralization of c-fos expression for: hemisphere $F_{(1,27)}=2.687, p=0.113$, partial $\eta^{2}=0.090^{\mathrm{u}}$; hemisphere $\times \operatorname{sex} F_{(1,27)}=0.073, p=0.788$, partial $\eta^{2}=$ $0.003^{v}$; hemisphere $\times$ condition $F_{(2,27)}=0.267, p=0.768$, partial $\eta^{2}=0.019^{\mathrm{w}}$; or hemisphere $\times$ sex $\times$ condition $F_{(2,27)}=0.473, p=0.628$, partial $\eta^{2}=0.034^{x}$.

As this demonstrated no lateralized effect of condition for $\mathrm{CRH}$ or c-fos expression, all further amygdala analyses were collapsed across hemispheres.

\section{Effects of sex: CRH and c-fos expression and colocalization}

There was a large difference in pattern of amygdala activation depending on the sex of the subject. In regards to amygdalar $\mathrm{CRH}$ expression, we observed a trend toward a significant main effect of sex $F_{(1,27)}=2.986, p=$ 0.095 , partial $\eta^{2}=0.100^{y}$, condition $F_{(2,27)}=2.601, p=$ 0.093 , partial $\eta^{2}=0.162^{z}$, and the interaction of sex $\times$ condition $F_{(2,27)}=2.677, p=0.087$, partial $\eta^{2}=0.165^{\text {aa }}$ on percentage difference in area expressing $\mathrm{CRH}$ in the amygdala. Indicating that males and females exhibited different patterns of amygdalar $\mathrm{CRH}$ expression in response to neonatal pain.

In our amygdalar c-fos measures, there was a significant main effect of $\operatorname{sex} F_{(1,27)}=6.86, p=0.014$, partial $\eta^{2}$ $=0.202^{\mathrm{ab}}$ in the percentage difference in area of the amygdala expressing c-fos: females (mean $=0.54 \%$, SEM $=10.98 \%$ ) males (mean $=84.22 \%$, SEM $=$ $27.80 \%)$. However, this was not the case for the effect of condition $F_{(2,27)}=2.48, p=0.103$, partial $\eta^{2}=0.155^{\mathrm{ac}}$, nor the interaction of sex $\times$ condition $F_{(2,27)}=1.63, p=$ 0.215 , partial $\eta^{2}=0.108^{\text {ad }}$ for percentage area of the amygdala expressing c-fos. Indicating that males and females exhibited different patterns of neuronal activity in response to neonatal pain.

Furthermore, there was a trend toward a significant main effect of $\operatorname{sex} F_{(1,26)}=3.44, p=0.075$, partial $\eta^{2}=$ $0.117^{\text {ae }}$ in the percentage difference in area expressing 
$\mathrm{CRH}$ in the hypothalamus. Suggesting that males and females exhibited different patterns of hypothalamic $\mathrm{CRH}$ expression in response to neonatal pain. This was not the case for the main effect of condition $F_{(2,26)}=0.022, p=$ 0.978 , partial $\eta^{2}=0.002^{\text {af }}$ nor the sex $\times$ condition interaction $F_{(2,26)}=0.883, p=0.426$, partial $\eta^{2}=0.064^{\text {ag }}$ for percentage difference in area expressing $\mathrm{CRH}$ in the hypothalamus. Additionally, we observed no effects or trends in the percentage difference in area expressing c-fos for the hypothalamus; $\operatorname{sex} F_{(1,26)}=0.132, p=0.720$, partial $\eta^{2}=0.005^{\text {ah }}$; condition $F_{(2,26)}=0.194, p=0.825$, partial $\eta^{2}=0.015^{\text {ai; }}$; and sex $\times$ condition $F_{(2,26)}=0.052, p$ $=0.949$, partial $\eta^{2}=0.004^{\mathrm{aj}}$.

There was a difference in the pattern of amygdala and hypothalamus c-fos and CRH mRNA expression depending on the sex of the subject. Preliminary $\mathrm{CRH}$ and c-fos colocalization analysis further exemplifies this sex difference. For amygdalar $\mathrm{CRH}$ and c-fos colocalization, there was a significant main effect of condition $F_{(2,27)}=3.958, p$ $=0.031$, partial $\eta^{2}=0.227^{\text {ak }}$. Subsequent Tukey's post hoc tests found that pain subjects (mean $=53$, SEM $=$ $5.62)$ had significantly more colocalization then undisturbed subjects (mean $=32.8$, SEM $=4.27$ ) $p=0.045 ; \mathrm{Cl}$ $=0.386-40.014^{\text {al }}$ and only trended toward significantly more than handled subjects (mean $=36.42$, SEM $=7.02$ ) $p=0.094 ; \mathrm{Cl}=-2.346-35.513^{\mathrm{am}}$. Additionally, there was a trend in the main effect of sex for amygdalar $\mathrm{CRH}$ and c-fos colocalization $F_{(1,27)}=3.348, p=0.078$, partial $\eta^{2}=0.110^{\text {an }}$, but not in the interaction of sex $\times$ condition $F_{(2,27)}=2.080, p=0.145$, partial $\eta^{2}=0.133^{\text {ao }}$. Further indicating that males and females react differently to neonatal adversity while also exemplifying differences between neonatal conditions.

There was no observed effects or interactions in the preliminary analysis of hypothalamic $\mathrm{CRH}$ and $\mathrm{c}$-fos colocalization; condition $F_{(2,26)}=0.155, p=0.857$, partial $\eta^{2}$ $=0.012^{\mathrm{ap}}$, sex $F_{(1,26)}=0.030, p=0.863$, partial $\eta^{2}=$ $0.001^{\text {aq }}$, sex $\times$ condition $F_{(2,26)}=0.413, p=0.666$, partial $\eta^{2}=0.031^{\text {ar }}$.

To conclude, our analysis of $\mathrm{CRH}$ and c-fos expression and colocalization within the amygdala and hypothalamus, exemplifies that $\mathrm{CRH}$ mRNA expression and neuronal activity (measured by c-fos mRNA expression) are sex dependent.

\section{Effects of neonatal pain on the amygdala}

Neonatal pain enhanced amygdalar $\mathrm{CRH}$ expression in male, but not female, subjects. There was a significant main effect of condition $F_{(2,13)}=4.93, p=0.026$, partial $\eta^{2}$ $=0.431^{\text {as }}$ in percentage difference in area expressing $\mathrm{CRH}$ in male amygdalar tissue (Fig. 6). Subsequent post hoc tests indicated a significant difference between male pain and undisturbed $(p=0.024 ; \mathrm{Cl} 8.52-123.84)^{\text {at }}$ but not handled subjects $(p=0.132 ; \mathrm{Cl}=-10.84-92.30)^{\text {au }}$. There was no observed effect of condition $F_{(2,14)}=0.658$, $p=0.533$, partial $\eta^{2}=0.086^{\mathrm{av}}$ in percentage difference in area expressing $\mathrm{CRH}$ in female amygdalar tissue.

Neonatal pain did not significantly affect amygdalar c-fos expression, despite an apparently similar trend with male $\mathrm{CRH}$ expression (Fig. 6). There was no observed effect of condition in percentage difference in area expressing c-fos for male $F_{(2,13)}=2.07, p=0.166$, partial $\eta^{2}$ $=0.241^{\text {aw }}$ or female $F_{(2,14)}=0.784, p=0.476$, partial $\eta^{2}$ $=0.101^{\mathrm{ax}}$ amygdala regions.

Neonatal pain increased amygdalar colocalization of $\mathrm{CRH}$ and c-fos in male, but not female, subjects. For male subjects, there was a significant main effect of condition $F_{(2,13)}=19.57, p<0.001$, partial $\eta^{2}=0.751^{\text {ay }}$ for amygdalar $\mathrm{CRH}$ and c-fos colocalization (Fig. 6). Subsequent post hoc tests indicated significantly higher levels of male amygdalar colocalization after pain compared to both handled $(p<0.001 ; \mathrm{Cl}=16.904-46.763)^{\mathrm{az}}$ and undisturbed conditions $(p=0.001 ; \mathrm{Cl}=14.642$ $48.025)^{\text {ba }}$. As was the case with $\mathrm{CRH}$ expression, females showed no significant effect of condition $F_{(2,14)}=0.429, p$ $=0.660$, partial $\eta^{2}=0.058^{\mathrm{bb}}$.

\section{Effects of neonatal pain on the hypothalamus}

Neonatal pain had minimal effects on hypothalamic expression of $\mathrm{CRH}, \mathrm{c}-\mathrm{fos}$, and its colocalization (Fig. 7). When separated by sex, neonatal pain did not significantly affect male $F_{(1,12)}=0.640, p=0.544$, partial $\eta^{2}=$ $0.096^{\mathrm{bc}}$; or female hypothalamic $\mathrm{CRH}$ expression $F_{(2,14)}=$ $0.341, p=0.717$, partial $\eta^{2}=0.046^{\text {bd }}$

Neonatal pain had no statistically significant effect on hypothalamic c-fos mRNA expression nor $\mathrm{CRH}$ and c-fos mRNA colocalization. Male hypothalamic c-fos expression $F_{(2,12)}=0.090, p=0.914$, partial $\eta^{2}=0.015^{\text {be }}$. The same was found for female hypothalamic c-fos expression $F_{(2,14)}=0.166, p=0.849$, partial $\eta^{2}=0.023^{\text {bf }}$. For hypothalamic $\mathrm{CRH}$ and c-fos colocalization, male colocalization: $F_{(2,12)}=0.113, p=0.894$, partial $\eta^{2}=0.019^{\text {bg }}$; and female colocalization: $F_{(2,14)}=0.684, p=0.521$, partial $\eta^{2}=0.089^{\text {bh }}$.

\section{Experiment 3: EPM and predator odor anxiety assays}

Outlier values were excluded from analysis that had a value that differed by $>2.5$ SDs from their respective group mean in any given measure (no $>2$ for any measure in any experimental group).

On PND24 and PND25, subjects were exposed to the EPM and predator odor testing in a counterbalanced order by day. As mentioned above (see Materials and Methods), due to methodological difficulties with the task (all subjects preferred the same side of the chamber, regardless of odor placement), predator odor data were not analyzed further. Nevertheless, it is still treated as an independent variable in our EPM experiment.

An initial 3 (condition: undisturbed, handled, pain) $\times 2$ (sex) $\times 2$ (order of testing; predator odor or EPM first) ANOVA was conducted on each dependent measure (reported below). As no significant differences due to sex or order of testing was found for any dependent measure, further analyses were conducted examining the effects of condition when collapsed across these variables.

\section{Effects of sex and testing order}

For open arm time, there was a nearly significant effect of condition $F_{(2,91)}=3.073, p=0.051$ partial $\eta^{2}=0.063^{\mathrm{bi}}$. There were no other significant effects or interactions. This includes the main effect of sex: $F_{(1,91)}=0.419, p=$ 
A
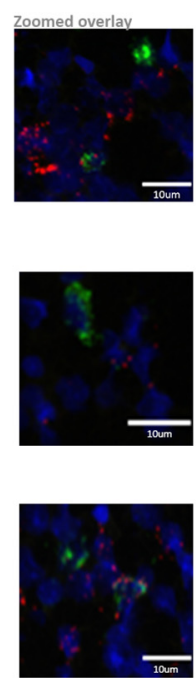

B
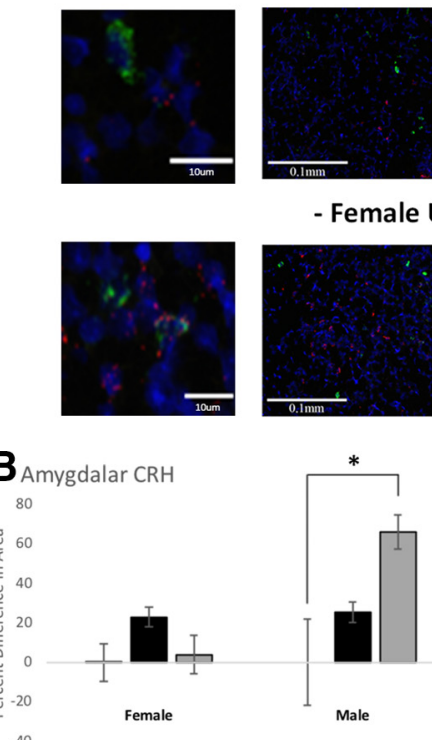

- Female Pain -

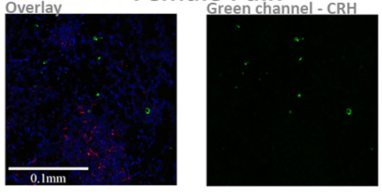

- Female Handle -

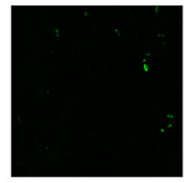

- Female Undisturbed -
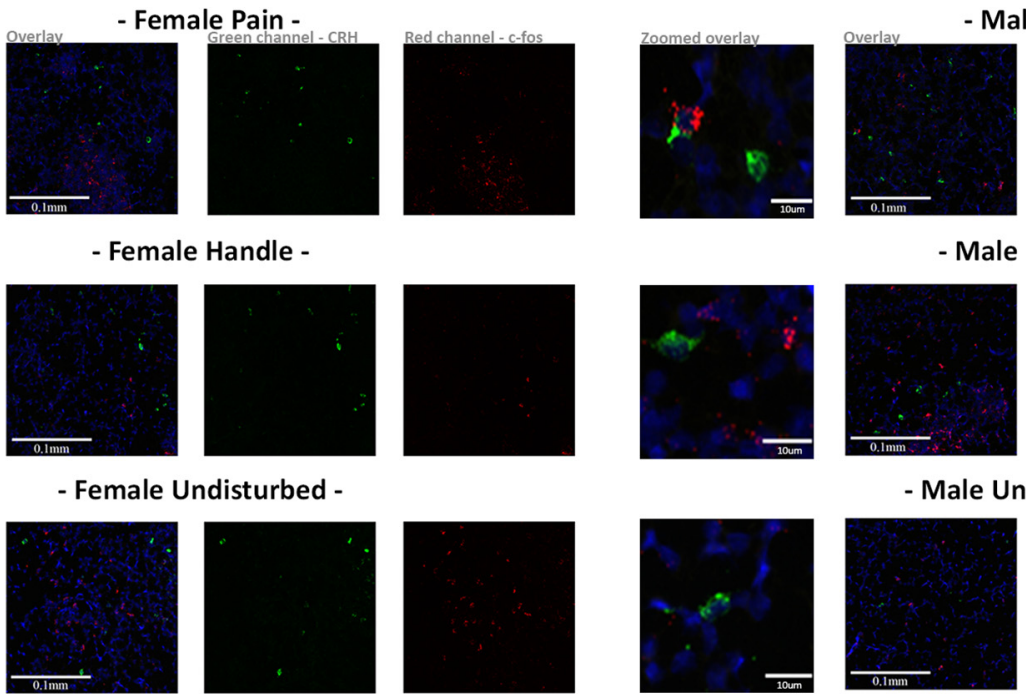

- Male Pain -
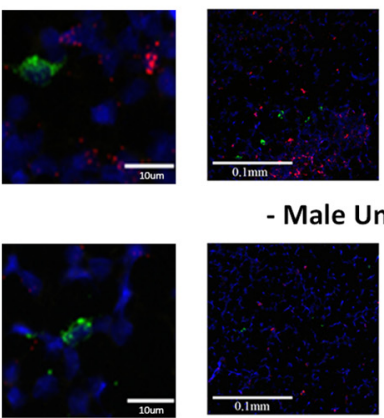

C Amygdalar c-fos

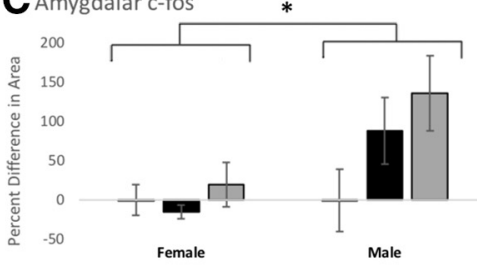

$-100$
D Amygdalar Colocalization

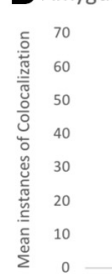

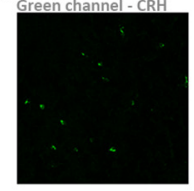

- Male Handle -
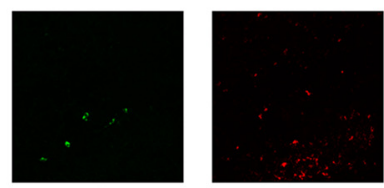

- Male Undisturbed -
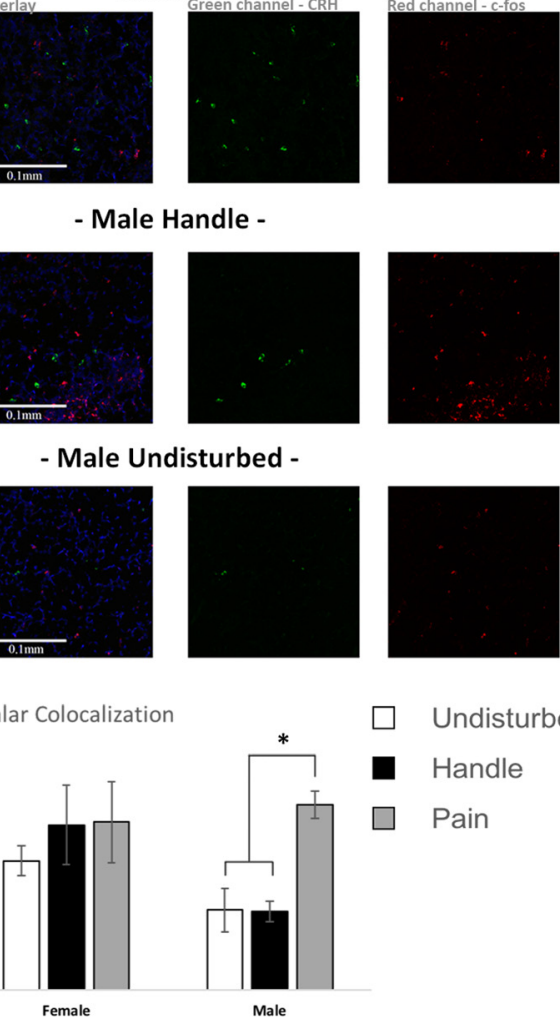

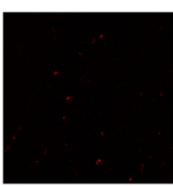

Undisturbed

Handle

Pain

Figure 6. Amygdalar quantification of FISH for transcription products $\mathrm{CRH}$ and c-fos. Examining the amygdala following acute neonatal manipulations [pain ( $N=5$ female, $N=6$ male), handled ( $N=6$ female, $N=6$ male), and undisturbed ( $N=6$ female, $N=$ 4 male)] in PND6 rats. $\boldsymbol{A}$, Representative amygdala segments for all neonatal manipulation groups with corresponding channel separation panels for $\mathrm{CRH}$ (green) and c-fos (red). DAPI was used as a counter stain for regional identification (blue). The first panel of each group is a zoomed snapshot of the corresponding overlay panel, whereas the next three panels represent a wider-field view of the overlay, $\mathrm{CRH}$ and $\mathrm{c}-\mathrm{fos}$, respectively. $\mathrm{B}, \mathrm{CRH}$ expression in handled and pain conditions as a percentage difference in area expressed from their same sex undisturbed counterpart. $\mathbf{C}, \mathbf{c}$-fos expression in handled and pain conditions as a percentage difference in area expressed from their same sex undisturbed counterpart. $\boldsymbol{D}$, colocalization of CRH and c-fos expression. For $\boldsymbol{B}-\boldsymbol{D}$, data are presented as means with error bars as \pm SEM; * denotes significant $(p<0.05)$ difference between indicated groups.

0.519 , partial $\eta^{2}=0.005^{\mathrm{bj}}$; and main effect of order: $F_{(1,91)}$ $=1.371, p=0.245$, partial $\eta^{2}=0.015^{\mathrm{bk}}$. There were also no significant interactions including sex $\times$ order: $F_{(1,91)}=$ $0.051, p=0.822$, partial $\eta^{2}=0.001^{\mathrm{bl}}$; sex $\times$ condition: $F_{(2,91)}=0.586, p=0.559$, partial $\eta^{2}=0.013^{\mathrm{bm}} ;$ order $\times$ condition: $F_{(2,91)}=0.141, p=0.868$, partial $\eta^{2}=0.003^{\text {bn }}$; and sex $\times$ order $\times$ condition: $F_{(2,91)}=0.533, p=0.588$, partial $\eta^{2}=0.012^{\mathrm{bo}}$.

For open arm entries, there were no significant effects or interactions. This includes the main effect of sex: $F_{(1,91)}=1.797, p=0.183$, partial $\eta^{2}=0.019^{\text {bp }}$; main effect of order: $F_{(1,91)}=1.527, p=0.220$, partial $\eta^{2}=0.017^{\mathrm{bq}}$; and main effect of condition: $F_{(2,91)}=$ $0.775, p=0.464$, partial $\eta^{2}=0.017^{\text {br }}$. There were also no significant interactions including sex $\times$ order: $F_{(1,91)}$ $=1.041, p=0.310$, partial $\eta^{2}=0.011^{\text {bs }}$; sex $\times$ condition: $F_{(2,91)}=0.580, p=0.562$, partial $\eta^{2}=0.013^{\text {bt }}$; order $\times$ condition: $F_{(2,91)}=0.745, p=0.478$, partial $\eta^{2}$ $=0.016^{\mathrm{bu}}$; and sex $\times$ order $\times$ condition: $F_{(2,91)}=1.198$, $p=0.307$, partial $\eta^{2}=0.026^{\text {bv }}$.

For total distance traveled there was a main effect of condition: $F_{(2,95)}=3.124, p=0.049$, partial $\eta^{2}=0.062^{\text {bw }}$, but no other significant effects or interactions. This in- cludes the main effect of sex: $F_{(1,95)}=1.839, p=0.178$, partial $\eta^{2}=0.019^{\text {bx }}$; and main effect of order: $F_{(1,95)}=$ $1.174, p=0.281$, partial $\eta^{2}=0.012^{\text {by }}$. There were also no significant interactions including sex $\times$ order: $F_{(1,95)}=$ $0.137, p=0.713$, partial $\eta^{2}=0.001^{\mathrm{bz}}$; sex $\times$ condition: $F_{(2,95)}=0.883, p=0.417$, partial $\eta^{2}=0.018^{\text {caa }}$; order $\times$ condition: $F_{(2,95)}=0.452, p=0.637$, partial $\eta^{2}=0.009^{\mathrm{cb}}$; and sex $\times$ order $\times$ condition: $F_{(2,95)}=1.134, p=0.326$, partial $\eta^{2}=0.023^{c c}$.

For total arm entries there was a trend toward a main effect of order: $F_{(1,92)}=3.259, p=0.074$, partial $\eta^{2}=$ $0.034^{\text {cd }}$, reflecting a tendency for previously stressed rats to make more transitions, but no other significant effects or interactions. This includes the main effect of sex: $F_{(1,92)}$ $=0.314, p=0.576$, partial $\eta^{2}=0.003^{\text {ce }}$; and main effect of condition: $F_{(2,92)}=1.748, p=0.180$, partial $\eta^{2}=$

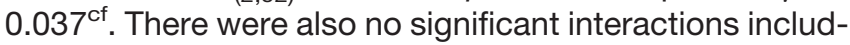
ing sex $\times$ order: $F_{(1,92)}=0.114, p=0.736$, partial $\eta^{2}$ $=0.001^{\mathrm{cg}}$; sex $\times$ condition: $F_{(2,92)}=0.943, p=0.363$, partial $\eta^{2}=0.020^{\text {ch. }}$; order $\times$ condition: $F_{(2,92)}=0.229, p$ $=0.795$, partial $\eta^{2}=0.005^{\mathrm{ci}}$; and sex $\times$ order $\times$ condition: $F_{(2,92)}=1.588, p=0.210$, partial $\eta^{2}=0.033^{\mathrm{cj}}$. 
A
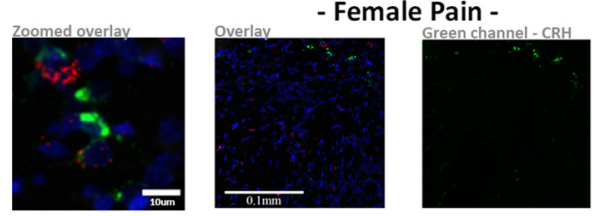

Female Handle -
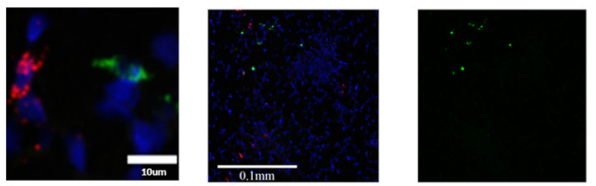

- Female Undisturbed -

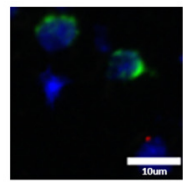

B

Hypothalamic CRH

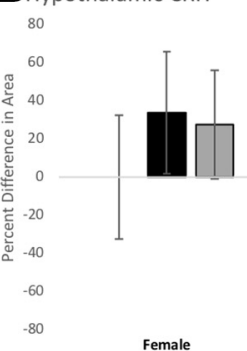

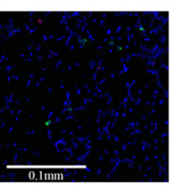

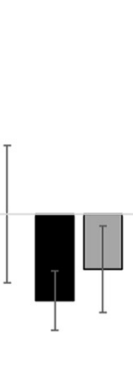

Male

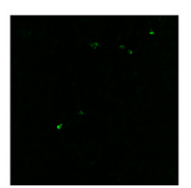

C Hypothalamic c-fos

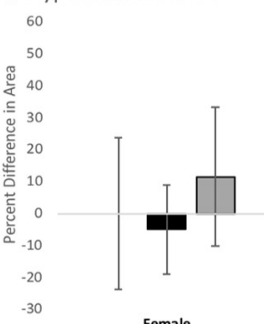

Female

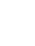
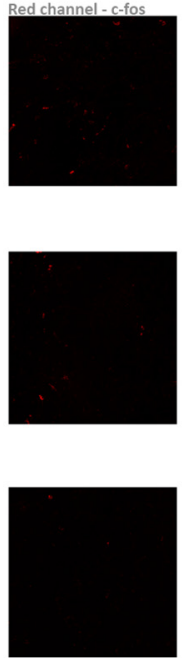

Ma
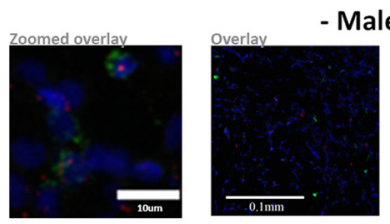

Male Pain -

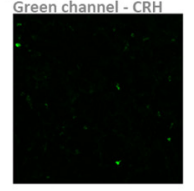

- Male Handle -
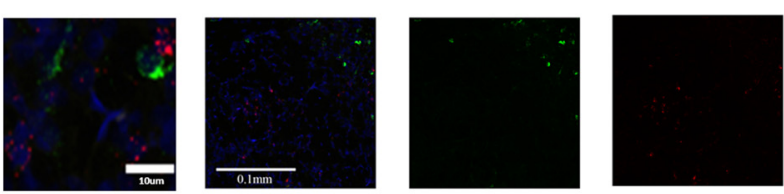

- Male Undisturbed -
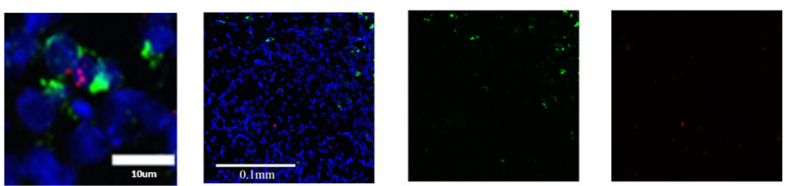

D

Hypothalamic Colocalization

Undisturbed
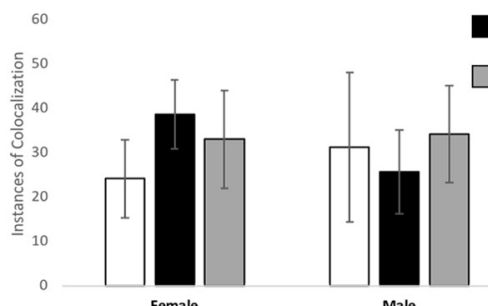

Handle

Pain

Figure 7. Hypothalamic quantification of FISH for transcription products $\mathrm{CRH}$ and c-fos. Examining hypothalamic expression following acute neonatal manipulations [pain ( $N=5$ female, $N=7$ male), handled $(N=6$ female, $N=4$ male), and undisturbed ( $N$ $=6$ female, $N=4$ male)] in PND6 rats. $\boldsymbol{A}$, Representative hypothalamus segments for all neonatal manipulation groups with corresponding channel separation panels for $\mathrm{CRH}$ (green) and c-fos (red). DAPI was used as a counter stain for regional identification (blue). The first panel of each group is a zoomed snapshot of the corresponding overlay panel, whereas the next three panels represent a wider-field view of the overlay, $\mathrm{CRH}$ and $\mathrm{c}$-fos, respectively. $\boldsymbol{B}, \mathrm{CRH}$ expression in handled and pain conditions as a percentage difference in area expressed from their same sex undisturbed counterpart. $\boldsymbol{C}, \mathbf{c}$-fos expression in handled and pain conditions as a percentage difference in area expressed from their same sex undisturbed counterpart. $\boldsymbol{D}$, Colocalization of $\mathrm{CRH}$ and c-fos expression. For $\boldsymbol{B}-\boldsymbol{D}$, data are presented as means with error bars as \pm SEM.

As no significant (or trending) effects of sex, testing order or breeding were found, all subsequent analyses were conducted as one-way ANOVAs examining the effects of condition.

\section{Effects of condition on elevated plus maze behavior}

When collapsed across the irrelevant variables, oneway ANOVAs reveal that neonatal pain affected time spent exploring open arms, as demonstrated by a significant effect of condition $F_{(2,100)}=3.49, p=0.034$, partial $\eta^{2}=0.065^{\mathrm{ck}}$ (Fig. 8). Tukey's post hoc tests demonstrate a significant difference between the neonatal pain and handled groups $(p=0.03 ; \mathrm{Cl}=23.33-0.72)^{\mathrm{cl}}$, but not between the pain and undisturbed groups $(p=0.15 ; \mathrm{Cl}=$ $-2.55-21.71)^{\mathrm{cm}}$ or the handled and undisturbed groups $\left(p=0.88 ; \mathrm{Cl}=-9.54-14.43^{\mathrm{cn}}\right.$; Fig. 8).

In addition, there was a trend toward an effect of condition on distance traveled $F_{(2,104)}=2.907, p=0.059$, partial $\eta^{2}=0.053^{\mathrm{co}}$ (Fig. 8), suggesting neonatal pain might produce a slight hyperactivity. There was no significant effect of condition on number of open arm entries $F_{(2,100)}=0.783, p=0.46$, partial $\eta^{2}=0.015^{\mathrm{cP}}$ or on total number of arm entries $F_{(2,101)}=1.847, p=0.163$, partial $\eta^{2}=0.035^{\mathrm{cq}}$.

\section{Discussion}

Our data show that repeated acute neonatal pain enhances amygdalar, but not hypothalamic, $\mathrm{CRH}$ expression in male, but not female, rats. In addition, we observed a decrease in later anxiety-like behavior that did not appear to be sex specific. Lastly, we observed no long-term differences in maternal rearing behaviors for litters subjected to neonatal manipulations. These data help clarify the mechanisms by which neonatal pain affects later behavior and provide insight into potential targets for future remediation.

Our first key finding is that during the neonatal period, male pups exposed to our acute pain manipulation exhibited higher levels of $\mathrm{CRH}$ expression in the amygdala compared to undisturbed (non-handled) controls. Additionally, we found that neonatal pain increases amygdalar $\mathrm{CRH}$ and c-fos mRNA colocalization compared to both handled and undisturbed controls. Our findings are the first to report enhanced amygdala $\mathrm{CRH}$ expression and 
A

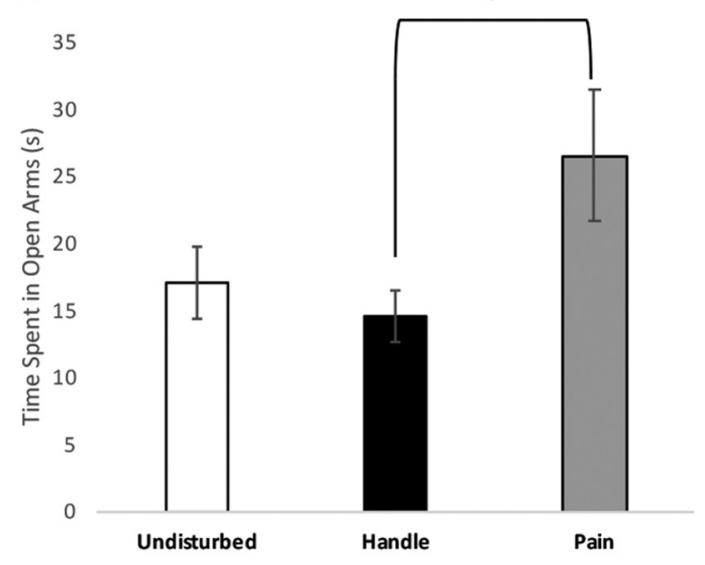

C

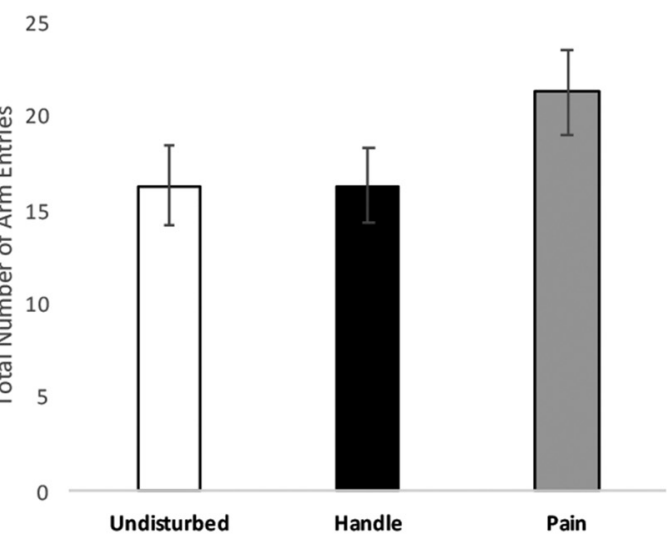

B

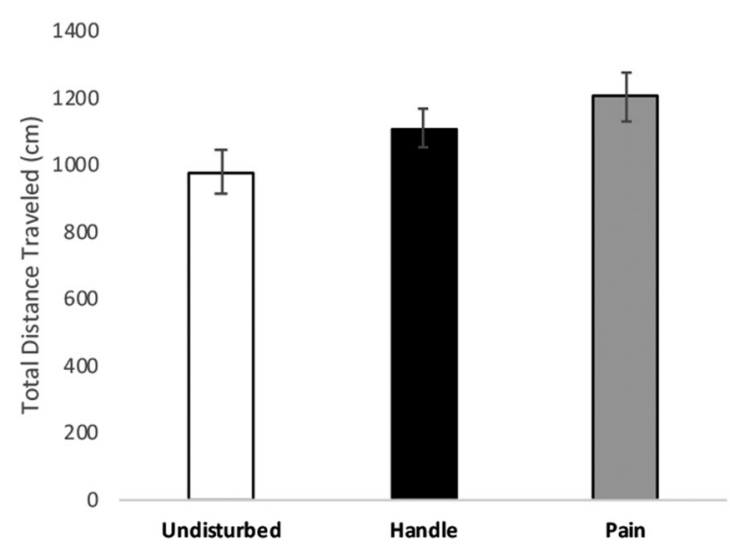

D

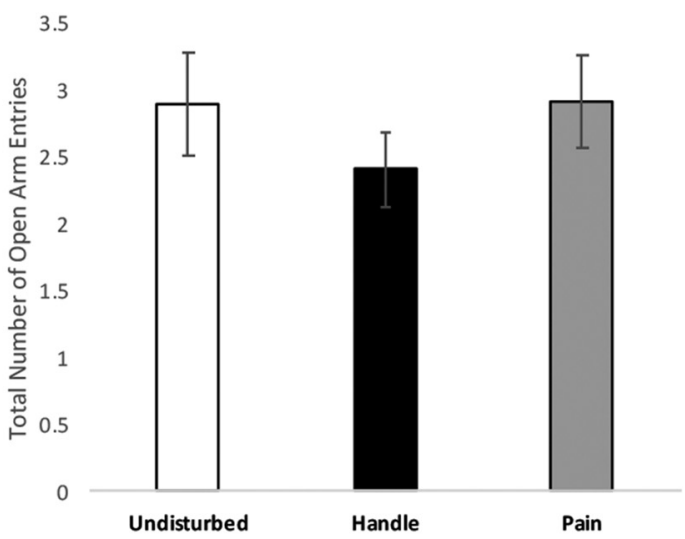

Figure 8. Effects of neonatal pain or handling on explorative and anxiety-like behavior in PND24 and PND25 rats using elevated plus maze anxiety test. Ns ranged from 29 to 38 per condition among all analyzed behaviors. The elevated plus maze data were measured and compared by $(\boldsymbol{A})$ time spent in open arms, $(\boldsymbol{B})$ total distance traveled, $(\boldsymbol{C})$ total number of arm entries, and $(\boldsymbol{D})$ total number of open arm entries. Data are presented as means with error bars as \pm SEM; * denotes significant $(p<0.05)$ difference between indicated groups.

enhanced c-fos expression, using a neonatal pain model. Nevertheless, these findings are consistent with previous work demonstrating that repeated instances of cold stress inflicted during the neonatal period increased amygdalar, but not hypothalamic, CRH mRNA expression (Hatalski et al., 1998). Additionally, in the adult literature, chronic painful stimuli has been shown to elevate amygdalar CRH expression (Ulrich-Lai et al., 2006). Although we were unable to find a significant difference in c-fos expression based on condition alone, our mRNA colocalization measure indicates that the amygdala is activated in response to our acute pain model. Adult rodent studies have also indicated that somatic nociception activates c-fos expression in the amygdala (Nakagawa et al., 2003). Thus, our data suggest that the neonatal amygdala may be reacting similarly to the adult amygdala in response to nociceptive stimuli.

This enhanced $\mathrm{CRH}$ expression was limited to males and only when compared to the undisturbed controls. Females did not show the same $\mathrm{CRH}$ expression pattern as males, in that there was not enhanced $\mathrm{CRH}$ or c-fos expression following neonatal pain in the amygdala. These findings are consistent with a prior study using only adult male rodents, which found that the amygdala but not hypothalamic $\mathrm{CRH}$ is enhanced following stress (Makino et al., 1999). The current data are also similar to other reports involving early life stress. For example, prenatally-stressed male rats exhibit higher levels of amygdala $\mathrm{CRH}-\mathrm{R} 1 \mathrm{mRNA}$ expression in the amygdala at two weeks old (PND13-PND14) compared to both male controls and experimental and control female groups (Brunton et al., 2011). Morphologic sex differences in rodent amygdala size has also been found in neonatally stressed PND20 males (Guadagno et al., 2018) and basal non-stressed adult males (Hines et al., 1992) both of which demonstrated an increase in amygdala size compared to respective female groups. Thus, the currently observed increase in amygdala CRH mRNA in only male rats may be reflective of a broader sex difference in the neurobiology of early-life stress. While the amygdala appears critical in males, other structures, such as the hypothalamus, may serve a similar function in females. For example, females have exhibited higher hypothalamic CRH mRNA expression within the first two weeks of life independent of prenatal stress conditions (Halasz et al., 1997). Additionally, PND14 females, but not males, 
showed a significant increase in hypothalamic $\mathrm{CRH}$ expression after exposure to prenatal stress (Halasz et al., 1997). In the present study, although there were apparent differences in the pattern of $\mathrm{CRH}$ mRNA expression in the hypothalamus between male and female subjects, we failed to find a significant effect in the hypothalamus of females which may reflect a lack of power (and therefore a Type II error) rather than a true negative result.

Our second key finding is that neonatal pain decreases anxiety-like behavior in juvenile rats. The EPM is a behavioral assay that has been validated to assess anxiety-like behavior in rodents, relying on a rodent's inclination toward enclosed spaces and avoidance of open spaces and heights (Walf and Frye, 2007). The current data demonstrate that early life pain increased time spent in open-arms in juvenile subjects. This has been reported several times in the literature. For example, rodents subjected to a single neonatal inflammatory injury displayed increased exploratory behaviors (in the EPM) in adulthood (Anseloni et al., 2005). In addition, non-painful tactile stimulation during the neonatal period exhibited an increase in later-life exploratory behaviors independent of sex in the EPM (Imanaka et al., 2008). Surprisingly, more tonic neonatal manipulations have resulted in no differences in later-life EPM behaviors when compared to both handled and undisturbed controls (Ranger et al., 2018). However, inconsistencies in rodent model (mouse versus rat) and manipulation procedure (4 vs 10 paw pricks daily) between the present study and the previous research likely contribute to these behavioral differences. Finally, acute neonatal pain (but not non-painful handling) manipulations have been shown to reduce laterlife fear expression following auditory fear conditioning (Davis et al., 2018). Thus, our findings are most consistent with the literature suggesting that neonatal pain may reduce subsequent fear and anxiety.

Thus, the current data demonstrate that neonatal pain both acutely elevates amygdalar $\mathrm{CRH}$ mRNA and causes a subsequent reduction in anxiety. One possible explanation linking these findings is that the high expression of amygdalar $\mathrm{CRH}$ during the neonatal period induces a later downregulation of amygdalar $\mathrm{CRH}$ signaling. This is consistent with the known physiology of $\mathrm{CRH}$ signaling (Dautzenberg and Hauger, 2002). Indeed, exposure to high levels of $\mathrm{CRH}$ causing receptor downregulation seems to be a critical component of the HPA-axis negative feedback loop (Aguilera, 1998). Previous work also shows that adult male rodents exhibit a downregulation of amygdalar $\mathrm{CRH}$ receptors in response to intracranial administration of $\mathrm{CRH}$ (Hauger et al., 1993). However, the developmental effects of high levels of $\mathrm{CRH}$ may be more complicated. Intracranial administration of $\mathrm{CRH}$ in neonatal rodents identified a subsequent upregulation of hippocampal and hypothalamic, but not amygdalar, $\mathrm{CRH} 1$ receptors (Brunson et al., 2002). Thus, the possibility that a compensatory decrease in amygdalar $\mathrm{CRH}$ signaling could be a driving force behind our observed juvenile anxiolytic responses needs more investigation.

The observed reduction in later-life anxiety resulting from neonatal adversity could be due, in part, to aspects of our neonatal manipulations that cause resilienceproducing changes in maternal behavior, rather than the pain per se. For example, it is well established that dams enhance pup grooming behaviors after litters are exposed to acute neonatal hind paw nociception (Smotherman et al., 1977; Walker et al., 2003) and rodent mothers enhance pup grooming behaviors immediately following 15-min bouts of maternal separation (Walker et al., 2008). These types of changes can mitigate against the effects of stress and trauma (Caldji et al., 1998; Walker et al., 2008; Kentner et al., 2019). Although our study did not find significant differences in maternal behaviors based on litter condition (neonatally manipulated, undisturbed), the current methodology was not designed to assess the acute effects of neonatal pain on maternal behavior, but to investigate whether there were enduring effects of the pain manipulation on dam-pup interactions, a fact which is not well established in the literature. Therefore, measurement occurred before the manipulations began on each day. In this regard, the current data demonstrate that there do not appear to be lasting consequences of neonatal pain on maternal behavior.

Although additional experiments are necessary to further flesh out potential sex differences in the effects of neonatal pain on hypothalamic $\mathrm{CRH}$ mRNA expression, the long-term behavioral consequences on other forms of anxiety (such as predator odor exposure), directly link the amygdala $\mathrm{CRH}$ system with later behavioral dysfunction, these studies yielded several novel findings. In particular, these data demonstrated that acute neonatal nociception affects early-life amygdala $\mathrm{CRH}$ mRNA expression in a sex dependent manner and reduced juvenile innate anxiety.

\section{References}

Aguilera G (1998) Corticotropin releasing hormone, receptor regulation and the stress response. Trends Endocrinol Metab 9:329336.

Anand KJS (2007) Pharmacological approaches to the management of pain in the neonatal intensive care unit. J Perinatol 27:S4-S11.

Anand KJS, Scalzo FM (2000) Can adverse neonatal experiences alter brain development and subsequent behavior? Biol Neonate 77:69-82

Anand KJ, Coskun V, Thrivikraman KV, Nemeroff CB, Plotsky PM (1999) Long-term behavioral effects of repetitive pain in neonatal rat pups. Physiol Behav 66:627-637.

Anseloni VCZ, He F, Novikova SI, Turnbach Robbins M, Lidow IA Ennis M, Lidow MS (2005) Alterations in stress-associated behaviors and neurochemical markers in adult rats after neonatal shortlasting local inflammatory insult. Neuroscience 131:635-645.

Bhutta A, Anand KJ (2001) Abnormal cognition and behavior in preterm neonates linked to smaller brain volumes. Trends Neurosci 24:129-130.

Bhutta AT, Cleves MA, Casey PH, Cradock MM, Anand KJS (2002) Cognitive and behavioral outcomes of school-aged children who were born preterm: a meta-analysis. JAMA 288:728-737.

Brunson KL, Avishai-Eliner S, Hatalski CG, Baram TZ (2001) Neurobiology of the stress response early in life: evolution of a concept and the role of corticotropin releasing hormone. Mol Psychiatry 6:647-656.

Brunson KL, Grigoriadis DE, Lorang MT, Baram TZ (2002) Corticotropin-releasing hormone $(\mathrm{CRH})$ downregulates the function of its receptor (CRF1) and induces CRF1 expression in hippocampal and cortical regions of the immature rat brain. Exp Neurol 176:75-86.

Brunton PJ, Donadio MVF, Russell JA (2011) Sex differences in prenatally programmed anxiety behaviour in rats: differential corticotropin-releasing hormone receptor mRNA expression in the amygdaloid complex. Stress 14:634-643.

Caldji C, Tannenbaum B, Sharma S, Francis D, Plotsky PM, Meaney MJ (1998) Maternal care during infancy regulates the development 
of neural systems mediating the expression of fearfulness in the rat. Proc Natl Acad Sci USA 95:5335-5340.

Carbajal R, Rousset A, Danan C, Coquery S, Nolent P, Ducroca S, Saizou C, Lapillonne A, Granier M, Durand P, Lenclen R, Coursol A, Hubert P, de Saint Blanquat L, Boëlle P-Y, Annequin D, Cimerman P, Anand KJS, Bréart G (2008) Epidemiology and treatment of painful procedures in neonates in intensive care units. JAMA 300:60-70.

Cratty MS, Ward HE, Johnson EA, Azzaro AJ, Birkle DL (1995) Prenatal stress increases corticotropin-releasing factor (CRF) content and release in rat amygdala minces. Brain Res 675:297-302.

Dautzenberg FM, Hauger RL (2002) The CRF peptide family and their receptors: yet more partners discovered. Trends Pharmacol Sci 23:71-77.

Davis M (1992) The role of the amygdala in fear and anxiety. Annu Rev Neurosci 15:353-375.

Davis SM, Rice M, Rudlong J, Eaton V, King T, Burman MA (2018) Neonatal pain and stress disrupts later-life Pavlovian fear conditioning and sensory function in rats: evidence for a two-hit model. Dev Psychobiol 60:520-533.

de Carvalho RC, Prado L, Rissardo Oliveira NC, Vilela Giusti FC, Santos Vieira J, Giusti-Paiva A, da Silva JRT, da Silva ML (2019) Repeated neonatal needle-prick stimulation increases inflammatory mechanical hypersensitivity in adult rats. Int J Dev Neurosci pii:S0736-5748(18)30346-0.

Fitzgerald M (1987) Cutaneous primary afferent properties in the hind limb of the neonatal rat. J Physiol 383:79-92.

Friard O, Gamba M (2016) BORIS: a free, versatile open-source event-logging software for video/audio coding and live observations. Methods Ecol Evol 7:1325-1330.

Grunau RE, Holsti L, Peters JWB (2006) Long-term consequences of pain in human neonates. Semin Fetal Neonatal Med 11:268-275.

Guadagno A, Wong TP, Walker C-D (2018) Morphological and functional changes in the preweaning basolateral amygdala induced by early chronic stress associate with anxiety and fear behavior in adult male, but not female rats. Prog Neuropsychopharmacol Biol Psychiatry 81:25-37.

Halasz I, Rittenhouse PA, Zorrilla EP, Redei E (1997) Sexually dimorphic effects of maternal adrenalectomy on hypothalamic corticotrophin-releasing factor, glucocorticoid receptor and anterior pituitary POMC mRNA levels in rat neonates. Brain Res Dev Brain Res 100:198-204.

Hatalski CG, Guirguis C, Baram TZ (1998) Corticotropin releasing factor mRNA expression in the hypothalamic paraventricular nucleus and the central nucleus of the amygdala is modulated by repeated acute stress in the immature rat. $J$ Neuroendocrinol 10:663-669.

Hauger RL, Irwin MR, Lorang M, Aguilera G, Brown MR (1993) High intracerebral levels of $\mathrm{CRH}$ result in $\mathrm{CRH}$ receptor downregulation in the amygdala and neuroimmune desensitization. Brain Res 616:283-292.

Hermann C, Hohmeister J, Demirakça S, Zohsel K, Flor H (2006) Long-term alteration of pain sensitivity in school-aged children with early pain experiences. Pain 125:278-285.

Hines M, Allen LS, Gorski RA (1992) Sex differences in subregions of the medial nucleus of the amygdala and the bed nucleus of the stria terminalis of the rat. Brain Res 579:321-326.

Holsboer F (1999) The rationale for corticotropin-releasing hormone receptor $(\mathrm{CRH}-\mathrm{R})$ antagonists to treat depression and anxiety. $\mathrm{J}$ Psychiatr Res 33:181-214.

Imanaka A, Morinobu S, Toki S, Yamamoto S, Matsuki A, Kozuru T, Yamawaki S (2008) Neonatal tactile stimulation reverses the effect of neonatal isolation on open-field and anxiety-like behavior, and pain sensitivity in male and female adult Sprague-Dawley rats. Behav Brain Res 186:91-97.

Janak PH, Tye KM (2015) From circuits to behaviour in the amygdala. Nature 517:284-292.

Kentner AC, Cryan JF, Brummelte S (2019) Resilience priming: translational models for understanding resiliency and adaptation to early life adversity. Dev Psychobiol 61:350-375.
Korte SM, De Boer SF (2003) A robust animal model of state anxiety: fear-potentiated behaviour in the elevated plus-maze. Eur J Pharmacol 463:163-175.

Kovács KJ (2008) Measurement of immediate-early gene activationc-fos and beyond. J Neuroendocrinol 20:665-672.

LaPrairie JL, Murphy AZ (2007) Female rats are more vulnerable to the long-term consequences of neonatal inflammatory injury. Pain 132 [Suppl 1]:S124-S133.

Makino S, Shibasaki T, Yamauchi N, Nishioka T, Mimoto T, Wakabayashi I, Gold PW, Hashimoto K (1999) Psychological stress increased corticotropin-releasing hormone mRNA and content in the central nucleus of the amygdala but not in the hypothalamic paraventricular nucleus in the rat. Brain Res 850:136-143.

Merali Z, Khan S, Michaud DS, Shippy SA, Anisman H (2004) Does amygdaloid corticotropin-releasing hormone $(\mathrm{CRH})$ mediate anxiety-like behaviors? Dissociation of anxiogenic effects and $\mathrm{CRH}$ release. Eur J Neurosci 20:229-239.

Nakagawa T, Katsuya A, Tanimoto S, Yamamoto J, Yamauchi Y, Minami M, Satoh M (2003) Differential patterns of c-fos mRNA expression in the amygdaloid nuclei induced by chemical somatic and visceral noxious stimuli in rats. Neurosci Lett 344:197-200.

Ogilvie KM, Rivier C (1997) Effect of alcohol on the proestrous surge of luteinizing hormone $(\mathrm{LH})$ and the activation of LH-releasing hormone (LHRH) neurons in the female rat. J Neurosci 17:2595-2604.

Plotsky PM, Thrivikraman KV, Nemeroff CB, Caldji C, Sharma S, Meaney MJ (2005) Long-term consequences of neonatal rearing on central corticotropin-releasing factor systems in adult male rat offspring. Neuropsychopharmacology 30:2192-2204.

Ranger M, Tremblay S, Chau CMY, Holsti L, Grunau RE, Goldowitz D (2018) Adverse behavioral changes in adult mice following neonatal repeated exposure to pain and sucrose. Front Psychol 9:2394-14.

Rincel M, Lépinay AL, Delage P, Fioramonti J, Théodorou VS, Layé S, Darnaudéry M (2016) Maternal high-fat diet prevents developmental programming by early-life stress. Transl Psychiatry 6:e966.

Schellinck HM, Stanford L, Darrah M (2003) Repetitive acute pain in infancy increases anxiety but does not alter spatial learning ability in juvenile mice. Behav Brain Res 142:157-165.

Schindelin J, Arganda-Carreras I, Frise E, Kaynig V, Longair M, Pietzsch T, Preibisch S, Rueden C, Saalfeld S, Schmid B, Tinevez JY, White DJ, Hartenstein V, Eliceiri K, Tomancak P, Cardona A (2012) Fiji: an open-source platform for biological-image analysis. Nat Methods 9:676-682.

Schneider CA, Rasband WS, Eliceiri KW (2012) NIH Image to ImageJ: 25 years of image analysis. Nat Methods 9:671-675.

Silveira MC, Sandner G, Graeff FG (1993) Induction of Fos immunoreactivity in the brain by exposure to the elevated plus-maze. Behav Brain Res 56:115-118.

Simons SHP, van Dijk M, Anand KS, Roofthooft D, van Lingen RA, Tibboel D (2003) Do we Still hurt newborn babies? A prospective study of procedural pain and analgesia in neonates. Arch Pediatr Adolesc Med 157:1058-1064.

Smotherman WP, Brown CP, Levine S (1977) Maternal responsiveness following differential pup treatment and mother-pup interactions. Horm Behav 8:242-253.

Ulrich-Lai YM, Xie W, Meij JTA, Dolgas CM, Yu L, Herman JP (2006) Limbic and HPA axis function in an animal model of chronic neuropathic pain. Physiol Behav 88:67-76.

Walf AA, Frye CA (2007) The use of the elevated plus maze as an assay of anxiety-related behavior in rodents. Nat Protoc 2:322-328.

Walker CD, Kudreikis K, Sherrard A, Johnston CC (2003) Repeated neonatal pain influences maternal behavior, but not stress responsiveness in rat offspring. Brain Res Dev Brain Res 140:253-261.

Walker CD, Xu Z, Rochford J, Johnston CC (2008) Naturally occurring variations in maternal care modulate the effects of repeated neonatal pain on behavioral sensitivity to thermal pain in the adult offspring. Pain 140:167-176. 